\title{
An exact estimate result for $p$-biharmonic equations with Hardy potential and negative exponents
}

\author{
Yanbin Sang $^{1 *}$ and Siman Guo ${ }^{1}$
}

\section{"Correspondence:}

sangyanbin@126.com

'Department of Mathematics,

School of Science, North University

of China, Taiyuan, P.R. China

\begin{abstract}
In this paper, $p$-biharmonic equations involving Hardy potential and negative exponents with a parameter $\lambda$ are considered. By means of the structure and properties of Nehari manifold, we give uniform lower bounds for $\Lambda>0$, which is the supremum of the set of $\lambda$. When $\lambda \in(0, \Lambda)$, the above problems admit at least two positive solutions.
\end{abstract}

Keywords: $p$-biharmonic equation; Nehari manifold; Positive solution; Negative exponents

\section{Introduction and preliminaries}

In this paper, we consider a $p$-biharmonic equation with Hardy potential and negative exponents:

$$
\begin{cases}\Delta_{p}^{2} u-\mu \frac{|u|^{p-2} u}{|x|^{2 p}}=f(x) u^{-q}+\lambda g(x) u^{\gamma} & \text { in } \Omega \backslash\{0\} \\ u(x)>0 & \text { in } \Omega \backslash\{0\} \\ u=\Delta u=0 & \text { on } \partial \Omega,\end{cases}
$$

where $0 \in \Omega \subset \mathbb{R}^{N}$ is a bounded smooth domain with $1<p<\frac{N}{2}, \Delta_{p}^{2} u=\Delta\left(|\Delta u|^{p-2} \Delta u\right)$ is the $p$-biharmonic operator. $\lambda>0$ is a parameter, $0<\mu<\mu_{N, p}=\left(\frac{(p-1) N(N-2 p)}{p^{2}}\right)^{p}, 0<q<1$ and $p-1<\gamma<p^{*}-1$, where $p^{*}=\frac{N p}{N-2 p}$ is called the critical Sobolev exponent. $f(x) \geq 0$, $f(x) \not \equiv 0, g(x)$ satisfies the requirement that the set $\{x \in \Omega: g(x)>0\}$ has positive measures, $\operatorname{supp} f \cap\{x \in \Omega: g(x)>0\} \neq \emptyset$ and $f, g \in C(\bar{\Omega})$. Biharmonic equations describe the sport of a rigid body and the deformations of an elastic beam. For example, this type of equation provides a model for considering traveling wave in suspension bridges $[5,16,27,30$, 36]. Various methods and tools have been adopted to deal with singular problems, such that fixed point theorems [14], topological methods [37], Fourier and Laurent transformation [18, 19], monotone iterative methods [21], global bifurcation theory [12], and degree theory $[22,31]$.

In recent years, there was much attention focused on the existence, multiplicity and qualitative properties of solutions for $p$-biharmonic equations under Dirichlet boundary conditions or Navier boundary conditions with Hardy terms [4, 15, 17, 32, 34]. Xie and

(c) The Author(s) 2019. This article is distributed under the terms of the Creative Commons Attribution 4.0 International License (http://creativecommons.org/licenses/by/4.0/), which permits unrestricted use, distribution, and reproduction in any medium, provided you give appropriate credit to the original author(s) and the source, provide a link to the Creative Commons license, and indicate if changes were made. 
Wang [32] studied the following $p$-biharmonic equation with Dirichlet boundary conditions:

$$
\begin{cases}\Delta_{p}^{2} u-\mu \frac{|u|^{p-2} u}{|x|^{2 p}}=f(x, u) & \text { in } \Omega \\ u=\frac{\partial u}{\partial n}=0 & \text { on } \partial \Omega\end{cases}
$$

where $\frac{\partial}{\partial n}$ is the outer normal derivative. By using the variational method, the existence of infinitely many solutions with positive energy levels for (1.2) was established. Huang and Liu [15] considered the following $p$-biharmonic equation with Navier boundary conditions:

$$
\begin{cases}\Delta_{p}^{2} u-\mu \frac{|u|^{p-2} u}{|x|^{2 p}}=f(x, u) & \text { in } \Omega \\ u=\Delta u=0 & \text { on } \partial \Omega\end{cases}
$$

where $1<p<\frac{N}{2}$. By using invariant sets of gradient flows, the authors proved that (1.3) possesses a sign-changing solution. Furthermore, Yang, Zhang and Liu [34] showed that (1.3) has a positive solution, a negative solution and a sequence of sign-changing solutions when $f$ satisfies appropriate conditions. Bhakta [4] established the qualitative properties of entire solutions for a noncompact problem related to $p$-biharmonic type equations with Hardy terms.

On the other hand, nonlinear biharmonic equations with negative exponents have been studied expensively $[1,6,8,13,20]$. Guerra [13] gave a complete description of entire radially symmetric solutions for the following biharmonic equation:

$$
\Delta^{2} u=-u^{-q}, \quad u>0 \quad \text { in } \mathbb{R}^{3},
$$

where $q>1$. Moreover, Cowan et al. [8] dealt with the regularity of the extremal solution of the following fourth order boundary value problems:

$$
\begin{cases}\Delta^{2} u=\frac{\lambda}{(1-u)^{2}} & \text { in } \Omega \\ 0<u<1 & \text { in } \Omega, \\ u=\frac{\partial u}{\partial n}=0 & \text { on } \partial \Omega .\end{cases}
$$

Very recently, Ansari, Vaezpour and Hesaaraki [1] considered fourth order elliptic problem with the combinations of Hardy term and negative exponents,

$$
\begin{cases}\Delta^{2} u-\lambda M\left(\|\nabla u\|^{2}\right) \Delta u-\frac{\mu}{|x|^{4}} u=\frac{h(x)}{u^{\gamma}}+k(x) u^{\alpha} & \text { in } \Omega, \\ u=\Delta u=0 & \text { on } \partial \Omega,\end{cases}
$$

where $\Omega \subset \mathbb{R}^{N}(N \geq 1)$ is a bounded $C^{4}$-domain, $\lambda$ and $\mu$ are positive parameters and $0<$ $\alpha<1,0<\gamma<1$ are constants. Here $M, h$ and $k$ are given continuous functions satisfying suitable hypotheses. By using the Galerkin method and the sharp angle lemma, the authors proved that problem (1.4) has a positive solution for $0<\mu<\left(\frac{N(N-4)}{4}\right)^{2}$. 
We say that $u \in W:=W^{2, p}(\Omega) \cap W_{0}^{1, p}(\Omega)$ is a weak solution of (1.1), if for every $\varphi \in W$, there holds

$$
\int_{\Omega}|\Delta u|^{p-2} \Delta u \Delta \varphi d x-\int_{\Omega} \frac{\mu}{|x|^{2 p}}|u|^{p-2} u \varphi d x=\int_{\Omega} f(x) u^{-q} \varphi d x+\lambda \int_{\Omega} g(x) u^{\gamma} \varphi d x .
$$

The following Rellich inequality will be used in this paper:

$$
\int_{\Omega}|\Delta u|^{p} d x \geq \mu_{N, p} \int_{\Omega} \frac{|u|^{p}}{|x|^{2 p}} d x, \quad \forall u \in W
$$

and it is not achieved $[9,24]$. For any $u \in W$, and $0<\mu<\mu_{N, p}$. The energy functional corresponding to (1.1) is defined by

$$
\begin{aligned}
I_{\lambda, \mu}(u)= & \frac{1}{p} \int_{\Omega}\left(|\Delta u|^{p}-\frac{\mu}{|x|^{2 p}}|u|^{p}\right) d x-\frac{1}{1-q} \int_{\Omega} f(x)|u|^{1-q} d x \\
& -\frac{\lambda}{\gamma+1} \int_{\Omega} g(x)|u|^{\gamma+1} d x .
\end{aligned}
$$

For $\mu \in\left[0, \mu_{N, p}\right), W$ is equipped with the following norm:

$$
\|u\|_{\mu}^{p}=\int_{\Omega}\left(|\Delta u|^{p}-\frac{\mu}{|x|^{2 p}}|u|^{p}\right) d x .
$$

Negative exponent term $u^{-q}$ implies that $I_{\lambda, \mu}$ is not differential on $W$, therefore, critical point theory cannot be applied to the problem (1.1) directly. We consider the following manifold:

$$
\mathcal{M}=\left\{u \in W:\|u\|_{\mu}^{p}=\int_{\Omega} f(x)|u|^{1-q} d x+\lambda \int_{\Omega} g(x)|u|^{\gamma+1} d x\right\},
$$

and make the following splitting for $\mathcal{M}$ :

$$
\begin{aligned}
& \mathcal{M}^{+}=\left\{u \in \mathcal{M}:(p+q-1)\|u\|_{\mu}^{p}>\lambda(\gamma+q) \int_{\Omega} g(x)|u|^{\gamma+1} d x\right\}, \\
& \mathcal{M}^{0}=\left\{u \in \mathcal{M}:(p+q-1)\|u\|_{\mu}^{p}=\lambda(\gamma+q) \int_{\Omega} g(x)|u|^{\gamma+1} d x\right\}, \\
& \mathcal{M}^{-}=\left\{u \in \mathcal{M}:(p+q-1)\|u\|_{\mu}^{p}<\lambda(\gamma+q) \int_{\Omega} g(x)|u|^{\gamma+1} d x\right\} .
\end{aligned}
$$

In this paper, we will study the dependence of problem (1.1) on $q, \gamma, f, g$ and $\Omega$ and evaluate the extremal value of $\lambda$ related to multiplicity of positive solutions for problem (1.1). Our idea comes from $[7,28,29]$. Our results improve and complement previous ones obtained in [23, 25]. Denote $\|u\|_{t}^{t}=\int_{\Omega}|u|^{t} d x$ and $D^{2, p}\left(\mathbb{R}^{N}\right)$ be the closure of $C_{0}^{\infty}\left(\mathbb{R}^{N}\right)$ with respect to the norm $\left(\int_{\mathbb{R}^{N}}|\Delta u|^{p} d x\right)^{\frac{1}{p}}$.

$\lambda_{1}$ denotes the smallest eigenvalue for

$$
\Delta_{p}^{2} u-\frac{\mu}{|x|^{2 p}}|u|^{p-2} u=\lambda_{1}|u|^{p-2} u, \quad x \in \Omega \backslash\{0\}, u \in W
$$


and $\varphi_{1}$ denotes the corresponding eigenfunction with $\varphi_{1}>0$ in $\Omega[3,10,26,33,35]$. The following minimization problem will be useful in the following discussions:

$$
S_{\mu}=\inf \left\{\int_{\mathbb{R}^{N}}\left(|\Delta u|^{p}-\frac{\mu}{|x|^{2 p}}|u|^{p}\right) d x, u \in D^{2, p}\left(\mathbb{R}^{N}\right), \int_{\mathbb{R}^{N}}|u|^{p^{*}} d x=1\right\}>0,
$$

and $S_{\mu}$ is achieved by a family of functions $[4,11]$. Thus, for every $u \in W \backslash\{0\},\|u\|_{p^{*}} \leq$ $\frac{\|u\|_{\mu}}{\sqrt[p]{S_{\mu}}}$. Therefore, combining with the Hölder inequality, we deduce that

$$
\begin{aligned}
\int_{\Omega}|u|^{\gamma+1} d x & \leq\left[\int_{\Omega}|u|^{(\gamma+1) \frac{p^{*}}{\gamma+1}} d x\right]^{\frac{\gamma+1}{p^{*}}}\left(\int_{\Omega} 1 d x\right)^{\frac{p^{*}-\gamma-1}{p^{*}}} \\
& =|\Omega|^{\frac{p^{*}-\gamma-1}{p^{*}}}\|u\|_{p^{*}}^{\gamma+1} \\
& \leq|\Omega|^{\frac{p^{*}-\gamma-1}{p^{*}}}\left(\frac{\|u\|_{\mu}}{\sqrt[p]{S_{\mu}}}\right)^{\gamma+1}, \\
\int_{\Omega}|u|^{1-q} d x & \leq\left[\int_{\Omega}|u|^{(1-q) \frac{p^{*}}{1-q}} d x\right]^{\frac{1-q}{p^{*}}}\left(\int_{\Omega} 1 d x\right)^{\frac{p^{*}-1+q}{p^{*}}} \\
& =|\Omega|^{\frac{p^{*}-1+q}{p^{*}}}\|u\|_{p^{*}}^{1-q} \\
& \leq|\Omega|^{\frac{p^{*}-1+q}{p^{*}}}\left(\frac{\|u\|_{\mu}}{\sqrt[p]{S_{\mu}}}\right)^{1-q},
\end{aligned}
$$

and

$$
\begin{aligned}
\int_{\Omega}|u|^{1-q} d x & \leq\left[\int_{\Omega}|u|^{(1-q) \frac{\gamma+1}{1-q}} d x\right]^{\frac{1-q}{\gamma+1}}\left(\int_{\Omega} 1 d x\right)^{\frac{\gamma+q}{\gamma+1}} \\
& =|\Omega|^{\frac{\gamma+q}{\gamma+1}}\|u\|_{\gamma+1}^{1-q} .
\end{aligned}
$$

Our main results are stated in the following theorems.

Theorem 1.1 Assume that $\lambda \in(0, \Lambda)$, where

$$
\begin{aligned}
\Lambda & \geq T_{\mu}=\left(\frac{q+p-1}{q+\gamma}\right)\left(\frac{\gamma-p+1}{q+\gamma}\right)^{\frac{p-\gamma-1}{1-q-p}}\left(\frac{1}{\|f\|_{\infty}}\right)^{\frac{p-\gamma-1}{1-q-p}}\left(\frac{1}{\|g\|_{\infty}}\right)\left(\frac{S_{\mu}}{|\Omega|^{\frac{p}{N}}}\right)^{\frac{q+\gamma}{p+q-1}} \\
& >0 .
\end{aligned}
$$

Then problem (1.1) admits at least two solutions $u_{0} \in \mathcal{M}^{+}, U_{0} \in \mathcal{M}^{-}$, with $\left\|U_{0}\right\|_{\mu}>\left\|u_{0}\right\|_{\mu}$.

Corollary 1.2 Let $U_{\lambda, \mu, \varepsilon} \in \mathcal{M}^{-}$be the solution of problem (1.1) with $\gamma=\varepsilon+p-1$, where $\lambda \in\left(0, T_{\mu}\right)$. Then

$$
\left\|U_{\lambda, \mu, \varepsilon}\right\|_{\mu}>C_{\mu, \varepsilon}\left(\frac{T_{\mu}}{\lambda}\right)^{\frac{1}{\varepsilon}}
$$


with

$$
C_{\mu, \varepsilon}=|\Omega|^{\frac{1}{p}}\left(\|f\|_{\infty}\right)^{\frac{1}{p+q-1}}\left(1+\frac{p+q-1}{\varepsilon}\right)^{\frac{1}{p+q-1}}\left(\frac{|\Omega|^{\frac{2}{N}}}{\sqrt[p]{S_{\mu}}}\right)^{\frac{1-q}{p+q-1}} \rightarrow \infty, \quad \text { as } \varepsilon \rightarrow 0 .
$$

Theorem 1.3 There exists $\lambda^{*}=\lambda^{*}(N, \Omega, \mu, q, \gamma)>0$ such that problem (1.1) with $f=g=1$ admits at least a positive solution for every $0<\lambda<\lambda^{*}$ and has no solution for every $\lambda>\lambda^{*}$.

\section{Some lemmas}

Lemma 2.1 Assume that $\lambda \in\left(0, T_{\mu}\right)$, where $T_{\mu}$ is defined in (1.15). Then $\mathcal{M}^{ \pm} \neq \emptyset$ and $\mathcal{M}^{0}=\{0\}$.

Proof (i) We can choose $u^{*} \in \mathcal{M} \backslash\{0\}$ such that $\int_{\Omega} f(x)\left|u^{*}\right|^{1-q} d x>0$ and $\int_{\Omega} g(x) \times$ $\left|u^{*}\right|^{\gamma+1} d x>0$ from the conditions imposed on $f$ and $g$. Denote

$$
\begin{aligned}
\varphi_{\mu}(t) & :=\frac{1}{t^{\gamma}} \frac{d}{d t} I_{\lambda, \mu}\left(t u^{*}\right) \\
& =t^{p-1-\gamma}\left\|u^{*}\right\|_{\mu}^{p}-t^{-q-\gamma} \int_{\Omega} f(x)\left|u^{*}\right|^{1-q} d x-\lambda \int_{\Omega} g(x)\left|u^{*}\right|^{\gamma+1} d x, \quad t>0 .
\end{aligned}
$$

Note that $\varphi_{\mu}^{\prime}(t)=(p-1-\gamma) t^{p-2-\gamma}\left\|u^{*}\right\|_{\mu}^{p}+(q+\gamma) t^{-1-q-\gamma} \int_{\Omega} f(x)\left|u^{*}\right|^{1-q} d x$. Let $\varphi_{\mu}^{\prime}(t)=0$, we have

$$
t:=t_{\max }=\left[\frac{(\gamma-p+1)\left\|u^{*}\right\|_{\mu}^{p}}{(q+\gamma) \int_{\Omega} f(x)\left|u^{*}\right|^{1-q} d x}\right]^{\frac{1}{1-q-p}}
$$

It is easy to check that $\varphi_{\mu}(t) \rightarrow-\infty$ as $t \rightarrow 0^{+}$and $\varphi_{\mu}(t) \rightarrow-\lambda \int_{\Omega} g(x)\left|u^{*}\right|^{\gamma+1} d x<0$ as $t \rightarrow \infty$. Furthermore, $\varphi_{\mu}(t)$ attains its maximum at $t_{\max }$. By (1.12) and (1.13), we obtain

$$
\begin{aligned}
\varphi_{\mu}\left(t_{\max }\right) & \\
= & {\left[\frac{(\gamma-p+1)\left\|u^{*}\right\|_{\mu}^{p}}{(q+\gamma) \int_{\Omega} f(x)\left|u^{*}\right|^{1-q} d x}\right]^{\frac{p-\gamma-1}{1-q-p}}\left\|u^{*}\right\|_{\mu}^{p} } \\
& -\left[\frac{(\gamma-p+1)\left\|u^{*}\right\|_{\mu}^{p}}{(q+\gamma) \int_{\Omega} f(x)\left|u^{*}\right|^{1-q} d x}\right]^{\frac{-q-\gamma}{1-q-p}} \int_{\Omega} f(x)\left|u^{*}\right|^{1-q} d x \\
& -\lambda \int_{\Omega} g(x)\left|u^{*}\right|^{\gamma+1} d x \\
= & \left(\frac{\gamma-p+1}{q+\gamma}\right)^{\frac{p-\gamma-1}{1-q-p}} \frac{\left(\left\|u^{*}\right\|_{\mu}^{p}\right)^{\frac{-\gamma-q}{1-q-p}}}{\left(\int_{\Omega} f(x)\left|u^{*}\right|^{1-q} d x\right)^{\frac{p-\gamma-1}{1-q-p}}} \\
& -\left(\frac{\gamma-p+1}{q+\gamma}\right)^{\frac{-q-\gamma}{1-q-p}} \frac{\left(\left\|u^{*}\right\|_{\mu}^{p}\right)^{\frac{-\gamma-q}{1-q-p}}}{\left(\int_{\Omega} f(x)\left|u^{*}\right|^{1-q} d x\right)^{\frac{p-\gamma-1}{1-q-p}}} \\
& -\lambda \int_{\Omega} g(x)\left|u^{*}\right|^{\gamma+1} d x \\
= & \left(\frac{q+p-1}{q+\gamma}\right)\left(\frac{\gamma-p+1}{q+\gamma}\right)^{\frac{p-\gamma-1}{1-q-p}} \frac{\left(\left\|u^{*}\right\|_{\mu}^{p}\right)^{\frac{-\gamma-q}{1-q-p}}}{\left(\int_{\Omega} f(x)\left|u^{*}\right|^{1-q} d x\right)^{\frac{p-\gamma-1}{1-q-p}}}-\lambda \int_{\Omega} g(x)\left|u^{*}\right|^{\gamma+1} d x
\end{aligned}
$$




$$
\begin{aligned}
& \geq\left(\frac{q+p-1}{q+\gamma}\right)\left(\frac{\gamma-p+1}{q+\gamma}\right)^{\frac{p-\gamma-1}{1-q-p}} \frac{\left(\left\|u^{*}\right\|_{\mu}^{p}\right)^{\frac{-\gamma-q}{1-q-p}}}{\left[\|f\|_{\infty}|\Omega|^{\frac{p^{*}-1+q}{p^{*}}}\left(\frac{\left\|u^{*}\right\|_{\mu}}{\sqrt[p]{S_{\mu}}}\right)^{1-q}\right]^{\frac{p-\gamma-1}{1-q-p}}} \\
& -\lambda\|g\|_{\infty}|\Omega|^{\frac{p^{*}-\gamma-1}{p^{*}}}\left(\frac{\left\|u^{*}\right\|_{\mu}}{\sqrt[p]{S_{\mu}}}\right)^{\gamma+1} \\
& =\left(\frac{q+p-1}{q+\gamma}\right)\left(\frac{\gamma-p+1}{q+\gamma}\right)^{\frac{p-\gamma-1}{1-q-p}}\left(\frac{1}{\|f\|_{\infty}}\right)^{\frac{p-\gamma-1}{1-q-p}} \frac{\left(\sqrt[p]{S_{\mu}}\right)^{\frac{(1-q)(p-\gamma-1)}{1-q-p}}}{|\Omega|^{\frac{p^{*}-1+q}{p^{*}} \frac{p-\gamma-1}{1-q-p}}}\left\|u^{*}\right\|_{\mu}^{\gamma+1} \\
& -\lambda\|g\|_{\infty}|\Omega|^{\frac{p^{*}-\gamma-1}{p^{*}}}\left(\frac{\left\|u^{*}\right\|_{\mu}}{\sqrt[p]{S_{\mu}}}\right)^{\gamma+1} \\
& =\left[\left(\frac{q+p-1}{q+\gamma}\right)\left(\frac{\gamma-p+1}{q+\gamma}\right)^{\frac{p-\gamma-1}{1-q-p}}\left(\frac{1}{\|f\|_{\infty}}\right)^{\frac{p-\gamma-1}{1-q-p}} \frac{\left(\sqrt[p]{S_{\mu}}\right)^{\frac{(1-q)(p-\gamma-1)}{1-q-p}}}{|\Omega|^{\frac{p^{*}-1+q}{p^{*}} \frac{p-\gamma-1}{1-q-p}}}\right. \\
& \left.-\lambda\|g\|_{\infty} \frac{|\Omega|^{\frac{p^{*}-\gamma-1}{p^{*}}}}{\left(\sqrt[p]{S_{\mu}}\right)^{\gamma+1}}\right]\left\|u^{*}\right\|_{\mu}^{\gamma+1} \\
& :=A(\mu, \lambda)\left\|u^{*}\right\|_{\mu}^{\gamma+1} \\
& >0 \text {. }
\end{aligned}
$$

When $A(\mu, \lambda)=0$, we get

$$
\begin{aligned}
\lambda & =\left(\frac{q+p-1}{q+\gamma}\right)\left(\frac{\gamma-p+1}{q+\gamma}\right)^{\frac{p-\gamma-1}{1-q-p}}\left(\frac{1}{\|f\|_{\infty}}\right)^{\frac{p-\gamma-1}{1-q-p}}\left(\frac{1}{\|g\|_{\infty}}\right) \frac{\left(\sqrt[p]{S_{\mu}}\right)^{\frac{(1-q)(p-\gamma-1)}{1-q-p}+\gamma+1}}{|\Omega|^{\frac{p^{*}-1+q}{p^{*}} \frac{p-\gamma-1}{1-q-p}+\frac{p^{*}-\gamma-1}{p^{*}}}} \\
& =\left(\frac{q+p-1}{q+\gamma}\right)\left(\frac{\gamma-p+1}{q+\gamma}\right)^{\frac{p-\gamma-1}{1-q-p}}\left(\frac{1}{\|f\|_{\infty}}\right)^{\frac{p-\gamma-1}{1-q-p}}\left(\frac{1}{\|g\|_{\infty}}\right)\left[\frac{S_{\mu}}{|\Omega|^{\frac{2 p}{N}}}\right]^{\frac{q+\gamma}{p+q-1}}=T_{\mu},
\end{aligned}
$$

where we use the following two equalities:

$$
\frac{(1-q)(p-\gamma-1)}{1-q-p}+\gamma+1=\frac{p(q+\gamma)}{q+p-1}
$$

and

$$
\frac{\left(p^{*}-1+q\right)(p-\gamma-1)}{p^{*}(1-q-p)}+\frac{p^{*}-\gamma-1}{p^{*}}=\frac{2 p(q+\gamma)}{N(q+p-1)} \text {. }
$$

In turn, this is also true. Hence $A(\mu, \lambda)=0$ if and only if $\lambda=T_{\mu}$. Thus for $\lambda \in\left(0, T_{\mu}\right)$, we have $A(\mu, \lambda)>0$. Moreover, by $(2.2)$, we derive that $\varphi_{\mu}\left(t_{\max }\right)>0$. Consequently, there exist two numbers $t_{\mu}^{-}$and $t_{\mu}^{+}$such that $0<t_{\mu}^{-}<t_{\max }<t_{\mu}^{+}$, and

$$
\varphi_{\mu}\left(t_{\mu}^{-}\right)=0=\varphi_{\mu}\left(t_{\mu}^{+}\right), \quad \varphi_{\mu}^{\prime}\left(t_{\mu}^{-}\right)>0>\varphi_{\mu}^{\prime}\left(t_{\mu}^{+}\right)
$$

It follows that $t_{\mu}^{-} u^{*} \in \mathcal{M}^{+}$, and $t_{\mu}^{+} u^{*} \in \mathcal{M}^{-}$. In fact, if $\varphi_{\mu}(t)=0$, then

$$
\varphi_{\mu}(t)=t^{p-1-\gamma}\|u\|_{\mu}^{p}-t^{-q-\gamma} \int_{\Omega} f(x)|u|^{1-q} d x-\lambda \int_{\Omega} g(x)|u|^{\gamma+1} d x=0,
$$


namely

$$
\|t u\|_{\mu}^{p}=\int_{\Omega} f(x)|t u|^{1-q} d x+\lambda \int_{\Omega} g(x)|t u|^{\gamma+1} d x .
$$

Hence $t u \in \mathcal{M}$. Furthermore, if $\varphi_{\mu}^{\prime}(t)>0$, then

$$
(p-1-\gamma) t^{p-2-\gamma}\|u\|_{\mu}^{p}+(q+\gamma) t^{-1-q-\gamma} \int_{\Omega} f(x)|u|^{1-q} d x>0
$$

That is

$$
(p-1-\gamma)\|t u\|_{\mu}^{p}+(q+\gamma) \int_{\Omega} f(x)|t u|^{1-q} d x>0
$$

i.e.,

$$
(p-1-\gamma)\|t u\|_{\mu}^{p}+(q+\gamma)\left[\|t u\|_{\mu}^{p}-\lambda \int_{\Omega} g(x)|t u|^{\gamma+1} d x\right]>0 .
$$

Note that $t u \in \mathcal{M}$, we have

$$
(p+q-1)\|t u\|_{\mu}^{p}-\lambda(q+\gamma) \int_{\Omega} g(x)|t u|^{\gamma+1} d x>0 .
$$

Thus $t u \in \mathcal{M}^{+}$. By a similar argument, if $\varphi_{\mu}(t)=0$ and $\varphi_{\mu}^{\prime}(t)<0$, then $t u \in \mathcal{M}^{-}$. Therefore, both $\mathcal{M}^{+}$and $\mathcal{M}^{-}$are non-empty sets for every $\lambda \in\left(0, T_{\mu}\right)$.

(ii) We claim that $\mathcal{M}^{0}=\{0\}$. Otherwise, we suppose that there exists $u_{*} \in \mathcal{M}^{0}$ and $u_{*} \neq 0$. Since $u_{*} \in \mathcal{M}^{0}$, we have

$$
(p+q-1)\left\|u_{*}\right\|_{\mu}^{p}=\lambda(\gamma+q) \int_{\Omega} g(x)\left|u_{*}\right|^{\gamma+1} d x
$$

moreover

$$
\begin{aligned}
0 & =\left\|u_{*}\right\|_{\mu}^{p}-\int_{\Omega} f(x) u_{*}^{1-q} d x-\lambda \int_{\Omega} g(x) u_{*}^{\gamma+1} d x \\
& =\left\|u_{*}\right\|_{\mu}^{p}-\int_{\Omega} f(x) u_{*}^{1-q} d x-\frac{p+q-1}{\gamma+q}\left\|u_{*}\right\|_{\mu}^{p} \\
& =\frac{\gamma-p+1}{\gamma+q}\left\|u_{*}\right\|_{\mu}^{p}-\int_{\Omega} f(x) u_{*}^{1-q} d x .
\end{aligned}
$$

For $\lambda \in\left(0, T_{\mu}\right)$ and $u_{*} \neq 0$, combining with (2.2), we deduce that

$$
\begin{aligned}
0 & <A(\mu, \lambda)\left\|u_{*}\right\|_{\mu}^{\gamma+1} \\
& \leq\left(\frac{q+p-1}{q+\gamma}\right)\left(\frac{\gamma-p+1}{q+\gamma}\right)^{\frac{p-\gamma-1}{1-q-p}} \frac{\left(\left\|u_{*}\right\|_{\mu}^{p}\right)^{\frac{-\gamma-q}{1-q-p}}}{\left(\frac{\gamma-p+1}{q+\gamma}\left\|u_{*}\right\|_{\mu}^{p}\right)^{\frac{p-\gamma-1}{1-q-p}}}-\left(\frac{q+p-1}{q+\gamma}\right)\left\|u_{*}\right\|_{\mu}^{p}=0,
\end{aligned}
$$

which is a contradiction, Thus $u_{*}=0$. That is, $\mathcal{M}^{0}=\{0\}$.

The gap structure in $\mathcal{M}$ is embodied in the following lemma. 
Lemma 2.2 Assume that $\lambda \in\left(0, T_{\mu}\right)$, then

$$
\begin{aligned}
& \|U\|_{\mu}>M_{\mu}(\lambda)>M_{\mu, 0}>\|u\|_{\mu}, \\
& \|U\|_{\gamma+1}>N_{\mu}(\lambda)>N_{\mu, 0}>\|u\|_{\gamma+1}, \quad \forall u \in \mathcal{M}^{+}, U \in \mathcal{M}^{-},
\end{aligned}
$$

where

$$
\begin{aligned}
& M_{\mu, 0}=\left[\frac{\gamma+q}{\gamma-p+1}\|f\|_{\infty} \frac{|\Omega|^{\frac{p^{*}-1+q}{p^{*}}}}{\left(\sqrt[p]{S_{\mu}}\right)^{1-q}}\right]^{\frac{1}{p+q-1}}, \\
& M_{\mu}(\lambda)=\left[\frac{p+q-1}{\lambda(\gamma+q)} \frac{1}{\|g\|_{\infty}} \frac{\left(\sqrt[p]{S_{\mu}}\right)^{\gamma+1}}{|\Omega|^{\frac{p^{*}-1-\gamma}{p^{*}}}}\right]^{\frac{1}{\gamma+1-p}}, \\
& N_{\mu, 0}=\left[\frac{\gamma+q}{\gamma-p+1}\|f\|_{\infty} \frac{|\Omega|^{\frac{\gamma+q}{\gamma+1}+\frac{\left(p^{*}-1-\gamma\right) p}{p^{*}(\gamma+1)}}}{S_{\mu}}\right]^{\frac{1}{p+q-1}}, \\
& N_{\mu}(\lambda)=\left[\frac{p+q-1}{\lambda(\gamma+q)} \frac{1}{\|g\|_{\infty}} \frac{S_{\mu}}{|\Omega|^{p\left(\frac{p^{*}-1-\gamma}{p^{*}(\gamma+1)}\right)}}\right]^{\frac{1}{\gamma+1-p}} .
\end{aligned}
$$

Proof If $u \in \mathcal{M}^{+} \subset \mathcal{M}$, then

$$
\begin{aligned}
0 & <(p+q-1)\|u\|_{\mu}^{p}-\lambda(\gamma+q) \int_{\Omega} g(x)|u|^{\gamma+1} d x \\
& =(p+q-1)\|u\|_{\mu}^{p}-(\gamma+q)\left[\|u\|_{\mu}^{p}-\int_{\Omega} f(x)|u|^{1-q} d x\right] \\
& =(p-\gamma-1)\|u\|_{\mu}^{p}+(\gamma+q) \int_{\Omega} f(x)|u|^{1-q} d x .
\end{aligned}
$$

We obtain from (1.13) that

$$
\begin{aligned}
(\gamma-p+1)\|u\|_{\mu}^{p} & <(\gamma+q) \int_{\Omega} f(x)|u|^{1-q} d x \\
& \leq(\gamma+q)\|f\|_{\infty}|\Omega|^{\frac{p^{*}-1+q}{p^{*}}}\left(\frac{\|u\|_{\mu}}{\sqrt[p]{S_{\mu}}}\right)^{1-q},
\end{aligned}
$$

which leads to

$$
\|u\|_{\mu}<\left[\frac{\gamma+q}{\gamma-p+1}\|f\|_{\infty} \frac{|\Omega|^{\frac{p^{*}-1+q}{p^{*}}}}{\left(\sqrt[p]{S_{\mu}}\right)^{1-q}}\right]^{\frac{1}{p+q-1}}=M_{\mu, 0} .
$$

By (1.12) and (1.14), we have

$$
\begin{aligned}
& (\gamma-p+1)\|u\|_{\gamma+1}^{p} \frac{S_{\mu}}{|\Omega|^{p\left(\frac{p^{*}-1-\gamma}{p^{*}(\gamma+1)}\right)}} \\
& \quad \leq(\gamma-p+1) \frac{S_{\mu}}{|\Omega|^{p \frac{p^{*}-1-\gamma}{p^{*}(\gamma+1)}}}\left[|\Omega|^{\frac{p^{*}-1-\gamma}{p^{*}}}\left(\frac{\|u\|_{\mu}}{\sqrt[p]{S_{\mu}}}\right)^{\gamma+1}\right]^{\frac{p}{\gamma+1}}
\end{aligned}
$$




$$
\begin{aligned}
& =(\gamma-p+1)\|u\|_{\mu}^{p} \\
& <(\gamma+q) \int_{\Omega} f(x)|u|^{1-q} d x \\
& \leq(\gamma+q)\|f\|_{\infty}|\Omega|^{\frac{\gamma+q}{\gamma+1}}\|u\|_{\gamma+1}^{1-q},
\end{aligned}
$$

which implies that

$$
\|u\|_{\gamma+1}<\left[\frac{\gamma+q}{\gamma-p+1}\|f\|_{\infty} \frac{|\Omega|^{\frac{\gamma+q}{\gamma+1}+\frac{\left(p^{*}-1-\gamma\right) p}{p^{*}(\gamma+1)}}}{S_{\mu}}\right]^{\frac{1}{p+q-1}}=N_{\mu, 0} .
$$

If $U \in \mathcal{M}^{-} \subset \mathcal{M}$, combining with (1.12), we derive that

$$
\begin{aligned}
(p+q-1)\|U\|_{\mu}^{p} & <\lambda(\gamma+q) \int_{\Omega} g(x)|U|^{\gamma+1} d x \\
& \leq \lambda(\gamma+q)\|g\|_{\infty}|\Omega|^{\frac{p^{*}-\gamma-1}{p^{*}}}\left(\frac{\|U\|_{\mu}}{\sqrt[p]{S_{\mu}}}\right)^{\gamma+1},
\end{aligned}
$$

which leads to

$$
\|U\|_{\mu}>\left[\frac{p+q-1}{\lambda(\gamma+q)} \frac{1}{\|g\|_{\infty}} \frac{\left(\sqrt[p]{S_{\mu}}\right)^{\gamma+1}}{|\Omega|^{\frac{p^{*}-1-\gamma}{p^{*}}}}\right]^{\frac{1}{\gamma+1-p}}=M_{\mu}(\lambda) .
$$

Furthermore

$$
\begin{aligned}
& (p+q-1)\|U\|_{\gamma+1}^{p} \frac{S_{\mu}}{|\Omega|^{p\left(\frac{p^{*}-1-\gamma}{p^{*}(\gamma+1)}\right)}} \\
& \leq(p+q-1) \frac{S_{\mu}}{|\Omega|^{\frac{p^{*}-1-\gamma}{p^{*}(\gamma+1)}}}\left[|\Omega|^{\frac{p^{*}-1-\gamma}{p^{*}}}\left(\frac{\|U\|_{\mu}}{\sqrt[p]{S_{\mu}}}\right)\right]^{\frac{p}{\gamma+1}} \\
& =(p+q-1)\|U\|_{\mu}^{p} \\
& <\lambda(\gamma+q) \int_{\Omega} g(x)|U|^{\gamma+1} d x \\
& \leq \lambda(\gamma+q)\|g\|_{\infty}\|U\|_{\gamma+1}^{\gamma+1},
\end{aligned}
$$

which means that

$$
\|U\|_{\gamma+1}>\left[\frac{p+q-1}{\lambda(\gamma+q)} \frac{1}{\|g\|_{\infty}} \frac{S_{\mu}}{|\Omega|^{p\left(\frac{p^{*}-1-\gamma}{p^{*}(\gamma+1)}\right)}}\right]^{\frac{1}{\gamma+1-p}}=N_{\mu}(\lambda) .
$$

Therefore

$$
\lambda=T_{\mu}=\left(\frac{q+p-1}{q+\gamma}\right)\left(\frac{\gamma-p+1}{q+\gamma}\right)^{\frac{p-\gamma-1}{1-q-p}}\left(\frac{1}{\|f\|_{\infty}}\right)^{\frac{p-\gamma-1}{1-q-p}}\left(\frac{1}{\|g\|_{\infty}}\right)\left(\frac{S_{\mu}}{|\Omega|^{\frac{2 p}{N}}}\right)^{\frac{q+\gamma}{p+q-1}}
$$




$$
\begin{aligned}
& \Leftrightarrow \quad M_{\mu}(\lambda)=\left[\frac{p+q-1}{\lambda(\gamma+q)} \frac{1}{\|g\|_{\infty}} \frac{\left(\sqrt[p]{S_{\mu}}\right)^{\gamma+1}}{|\Omega|^{\frac{p^{*}-1-\gamma}{p^{*}}}}\right]^{\frac{1}{\gamma+1-p}} \\
& =\lambda^{-\frac{1}{\gamma+1-p}}\left[\frac{p+q-1}{\gamma+q} \frac{1}{\|g\|_{\infty}} \frac{\left(\sqrt[p]{S_{\mu}}\right)^{\gamma+1}}{|\Omega|^{\frac{p^{*}-1-\gamma}{p^{*}}}}\right]^{\frac{1}{\gamma+1-p}} \\
& =\left(\frac{q+\gamma}{q+p-1}\right)^{\frac{1}{\gamma+1-p}}\left(\frac{q+\gamma}{\gamma-p+1}\right)^{\frac{1}{p+q-1}}\left(\|f\|_{\infty}\right)^{\frac{1}{p+q-1}}\left(\|g\|_{\infty}\right)^{\frac{1}{\gamma+1-p}} \\
& \times \frac{|\Omega|^{\frac{2 p}{N}} \frac{q+\gamma}{(q+p-1)(\gamma+1-p)}}{\left(S_{\mu}\right)^{\frac{q+\gamma}{(p+q-1)(\gamma+1-p)}}}\left[\frac{p+q-1}{\gamma+q} \frac{1}{\|g\|_{\infty}} \frac{\left(\sqrt[p]{S_{\mu}}\right)^{\gamma+1}}{|\Omega|^{\frac{p^{*}-1-\gamma}{p^{*}}}}\right]^{\frac{1}{\gamma+1-p}} \\
& =\left(\frac{q+\gamma}{\gamma-p+1}\right)^{\frac{1}{p+q-1}}\left(\|f\|_{\infty}\right)^{\frac{1}{p+q-1}} \frac{|\Omega|^{\frac{2 p}{N} \frac{q+\gamma}{(\gamma-p+1)(p+q-1)}-\frac{p^{*}-1-\gamma}{p^{*}}}}{\left(\sqrt[p]{S_{\mu}}\right)^{p \frac{q+\gamma}{(\gamma-p+1)(p+q-1)}-\frac{\gamma+1}{\gamma+1-p}}} \\
& =\left[\frac{\gamma+q}{\gamma-p+1}\|f\|_{\infty} \frac{|\Omega|^{\frac{p^{*}-1+q}{p^{*}}}}{\left(\sqrt[p]{S_{\mu}}\right)^{1-q}}\right]^{\frac{1}{p+q-1}}=M_{\mu, 0}
\end{aligned}
$$

where we have used the following facts:

$$
\begin{aligned}
\frac{2 p}{N} & \frac{q+\gamma}{(\gamma-p+1)(p+q-1)}-\frac{p^{*}-1-\gamma}{p^{*}(\gamma-p+1)} \\
& =\frac{2 p\left(p^{*}-p\right)}{2 p p^{*}} \frac{q+\gamma}{(\gamma-p+1)(p+q-1)}-\frac{p^{*}-1-\gamma}{p^{*}(\gamma-p+1)} \\
& =\frac{(\gamma-p+1)\left(p^{*}+q-1\right)}{p^{*}(\gamma-p+1)(p+q-1)},
\end{aligned}
$$

and

$$
p \frac{q+\gamma}{(\gamma-p+1)(p+q-1)}-\frac{\gamma+1}{\gamma+1-p}=\frac{p q-q \gamma+\gamma-p-q+1}{(\gamma-p+1)(p+q-1)}=\frac{1-q}{p+q-1} \text {. }
$$

Similarly

$$
\begin{aligned}
& \lambda=T_{\mu}=\left(\frac{q+p-1}{q+\gamma}\right)\left(\frac{\gamma-p+1}{q+\gamma}\right)^{\frac{p-\gamma-1}{1-q-p}}\left(\frac{1}{\|f\|_{\infty}}\right)^{\frac{p-\gamma-1}{1-q-p}}\left(\frac{1}{\|g\|_{\infty}}\right)\left[\frac{S_{\mu}}{|\Omega|^{\frac{2 p}{N}}}\right]^{\frac{q+\gamma}{p+q-1}} . \\
& \Leftrightarrow \quad N_{\mu}(\lambda)=\left[\frac{p+q-1}{\lambda(\gamma+q)} \frac{1}{\|g\|_{\infty}} \frac{S_{\mu}}{|\Omega|^{p\left(\frac{p^{*}-1-\gamma}{p^{*}(\gamma+1)}\right)}}\right]^{\frac{1}{\gamma+1-p}} \\
& =\lambda^{-\frac{1}{\gamma+1-p}}\left[\frac{p+q-1}{\lambda(\gamma+q)} \frac{1}{\|g\|_{\infty}} \frac{S_{\mu}}{|\Omega|^{p\left(\frac{p^{*}-1-\gamma}{p^{*}(\gamma+1)}\right)}}\right]^{\frac{1}{\gamma+1-p}} \\
& =\left(\frac{q+\gamma}{q+p-1}\right)^{\frac{1}{\gamma+1-p}}\left(\frac{q+\gamma}{\gamma-p+1}\right)^{\frac{1}{p+q-1}}\left(\|f\|_{\infty}\right)^{\frac{1}{p+q-1}}\left(\|g\|_{\infty}\right)^{\frac{1}{\gamma+1-p}} \\
& \times \frac{|\Omega|^{\frac{2 p}{N} \frac{q+\gamma}{(q+p-1)(\gamma+1-p)}}}{\left(S_{\mu}\right)^{\frac{q+\gamma}{(p+q-1)(\gamma+1-p)}}}\left[\frac{p+q-1}{\lambda(\gamma+q)} \frac{1}{\|W\|_{\infty}} \frac{S_{\mu}}{|\Omega|^{p\left(\frac{p^{*}-1-\gamma}{p^{*}(\gamma+1)}\right)}}\right]^{\frac{1}{\gamma+1-p}}
\end{aligned}
$$




$$
\begin{aligned}
& =\left(\frac{q+\gamma}{\gamma-p+1}\right)^{\frac{1}{p+q-1}}\left(\|f\|_{\infty}\right)^{\frac{1}{p+q-1}|\Omega|^{\frac{2 p}{N} \frac{q+\gamma}{(\gamma-p+1)(p+q-1)}-p} \frac{p^{*}-1-\gamma}{p^{*}(\gamma+1)(\gamma+1-p)}} \\
& \left(S_{\mu}\right)^{\frac{q+\gamma}{(\gamma-p+1)(p+q-1)}-\frac{1}{\gamma+1-p}} \\
& =\left[\frac{\gamma+q}{\gamma-p+1}\|f\|_{\infty} \frac{|\Omega|^{\frac{\gamma+q}{\gamma+1}+\frac{\left(p^{*}-1-\gamma\right) p}{p^{*}(\gamma+1)}}}{S_{\mu}}\right]^{\frac{1}{p+q-1}}=N_{\mu, 0},
\end{aligned}
$$

where we have applied the following equalities:

$$
\begin{aligned}
& \frac{2 p}{N} \frac{q+\gamma}{(\gamma-p+1)(p+q-1)}-p \frac{p^{*}-1-\gamma}{p^{*}(\gamma+1)(\gamma+1-p)} \\
& \quad=\frac{2 p\left(p^{*}-p\right)}{2 p p^{*}} \frac{q+\gamma}{(\gamma-p+1)(p+q-1)}-\frac{p^{*}-1-\gamma}{p^{*}(\gamma-p+1)} \\
& \quad=\frac{\gamma+q}{\gamma+1}+p \frac{p^{*}-1-\gamma}{p^{*}(\gamma+1)},
\end{aligned}
$$

and

$$
\frac{q+\gamma}{(\gamma-p+1)(p+q-1)}-\frac{1}{\gamma+1-p}=\frac{q+\gamma-(p+q-1)}{(\gamma-p+1)(p+q-1)}=\frac{1}{p+q-1} .
$$

Consequently, $M_{\mu}(\lambda)=M_{\mu, 0}$ if and only if $\lambda=T_{\mu}$ and $N_{\mu}(\lambda)=N_{\mu, 0}$ if and only if $\lambda=T_{\mu}$ respectively. This completes the proof of Lemma 2.2.

Lemma 2.3 Assume that $\lambda \in\left(0, T_{\mu}\right)$. Then $\mathcal{M}^{-}$is a closed set in W-topology.

Proof We choose a sequence $\left\{U_{n}\right\}$ such that $\left\{U_{n}\right\} \subset \mathcal{M}^{-}$and $U_{n} \rightarrow U_{0}$ with $U_{0} \in W$. Then

$$
\begin{aligned}
\left\|U_{0}\right\|_{\mu}^{p} & =\lim _{n \rightarrow \infty}\left\|U_{n}\right\|_{\mu}^{p} \\
& =\lim _{n \rightarrow \infty}\left[\int_{\Omega} f(x)\left|U_{n}\right|^{1-q} d x+\lambda \int_{\Omega} g(x)\left|U_{n}\right|^{\gamma+1} d x\right] \\
& =\int_{\Omega} f(x)\left|U_{0}\right|^{1-q} d x+\lambda \int_{\Omega} g(x)\left|U_{0}\right|^{\gamma+1} d x,
\end{aligned}
$$

and

$$
\begin{aligned}
(p & +q-1)\left\|U_{0}\right\|_{\mu}^{p}-\lambda(\gamma+q) \int_{\Omega} g(x)\left|U_{0}\right|^{\gamma+1} d x \\
& =\lim _{n \rightarrow \infty}\left[(p+q-1)\left\|U_{n}\right\|_{\mu}^{p}-\lambda(\gamma+q) \int_{\Omega} g(x)\left|U_{n}\right|^{\gamma+1} d x\right] \leq 0 .
\end{aligned}
$$

Hence $U_{0} \in \mathcal{M}^{-} \cup \mathcal{M}^{0}$. By Lemma 2.2, we have

$$
\left\|U_{0}\right\|_{\mu}=\lim _{n \rightarrow \infty}\left\|U_{n}\right\|_{\mu} \geq M_{\mu, 0}>0
$$

that is, $U_{0} \neq 0$. Combining with Lemma 2.1 , we obtain $U_{0} \notin \mathcal{M}^{0}$. Thus $U_{0} \in \mathcal{M}^{-}$. Therefore $\mathcal{M}^{-}$is a closed set in $W$-topology for every $\lambda \in\left(0, T_{\mu}\right)$. 
Lemma 2.4 For $u \in \mathcal{M}^{ \pm}$, there exist a number $\varepsilon>0$ and a continuous function $\widetilde{g}(h)>0$ with $h \in W$ and $\|h\|<\varepsilon$ such that

$$
\tilde{g}(0)=1, \quad \tilde{g}(h)(u+h) \in \mathcal{M}^{ \pm}, \quad \forall h \in W,\|h\|<\varepsilon .
$$

Proof We only prove the case that $\mathcal{M}^{+}$. Define a function $\widetilde{F}: W \times \mathbb{R}^{+} \rightarrow \mathbb{R}$ by:

$$
\widetilde{F}(h, s)=s^{p-1+q}\|u+h\|_{\mu}^{p}-\int_{\Omega} f(x)|u+h|^{1-q} d x-\lambda s^{\gamma+q} \int_{\Omega} g(x)|u+h|^{\gamma+1} d x .
$$

Note that $u \in \mathcal{M}^{+}$, we obtain

$$
\widetilde{F}(0,1)=\|u\|_{\mu}^{p}-\int_{\Omega} f(x)|u|^{1-q} d x-\lambda \int_{\Omega} g(x)|u|^{\gamma+1} d x=0,
$$

and

$$
\widetilde{F}_{s}(0,1)=(p-1+q)\|u\|_{\mu}^{p}-(q+\gamma) \lambda \int_{\Omega} g(x)|u|^{\gamma+1} d x>0 .
$$

At $(0,1)$, using the implicit function theorem, we know that there exists $\bar{\varepsilon}>0$ such that for $h \in W$ and $\|h\|<\bar{\varepsilon}$, the equation $\widetilde{F}(h, s)=0$ has a unique continuous solution $s=\widetilde{g}(h)>0$. Hence $\tilde{g}(0)=1$ and

$$
\begin{aligned}
0 & =\widetilde{g}(h)^{p-1+q}\|u+h\|_{\mu}^{p}-\int_{\Omega} f(x)|u+h|^{1-q} d x-\lambda \widetilde{g}(h)^{\gamma+q} \int_{\Omega} g(x)|u+h|^{\gamma+1} d x \\
& =\frac{\|\widetilde{g}(h)(u+h)\|_{\mu}^{p}-\int_{\Omega} f(x)|\widetilde{g}(h)(u+h)|^{1-q} d x-\lambda \int_{\Omega} g(x)|\widetilde{g}(h)(u+h)|^{\gamma+1} d x}{\widetilde{g}(h)^{1-q}},
\end{aligned}
$$

i.e.,

$$
\widetilde{g}(h)(u+h) \in \mathcal{M}, \quad \forall h \in W,\|h\|<\bar{\varepsilon},
$$

and

$$
\begin{aligned}
\widetilde{F}_{s}(h, \widetilde{g}(h)) & =(p-1+q) \widetilde{g}(h)^{p+q-2}\|u+h\|_{\mu}^{p}-(q+\gamma) \lambda \widetilde{g}(h)^{\gamma+q-1} \int_{\Omega} g(x)|u+h|^{\gamma+1} d x \\
& =\frac{(p-1+q)\|\widetilde{g}(h)(u+h)\|_{\mu}^{p}-(q+\gamma) \lambda \int_{\Omega} g(x)|\widetilde{g}(h)(u+h)|^{\gamma+1} d x}{\widetilde{g}^{2-q}(h)},
\end{aligned}
$$

together with (2.3), these imply that we can choose $\varepsilon>0$ small enough $(\varepsilon<\bar{\varepsilon})$ such that for every $h \in W$ and $\|h\|<\varepsilon$

$$
(p-1+q)\|\widetilde{g}(h)(u+h)\|_{\mu}^{p}-(q+\gamma) \lambda \int_{\Omega} g(x)|\widetilde{g}(h)(u+h)|^{\gamma+1} d x>0,
$$

that is,

$$
\widetilde{g}(h)(u+h) \in \mathcal{M}^{+}, \quad \forall h \in W,\|h\|<\varepsilon .
$$

This completes the proof of Lemma 2.3. 


\section{Proof of Theorem 1.1}

For every $u \in \mathcal{M}$, by (1.13), we have

$$
\begin{aligned}
I_{\lambda, \mu}(u) & =\frac{1}{p}\|u\|_{\mu}^{p}-\frac{1}{1-q} \int_{\Omega} f(x)|u|^{1-q} d x-\frac{\lambda}{\gamma+1} \int_{\Omega} g(x)|u|^{\gamma+1} d x \\
& =\frac{1}{p}\|u\|_{\mu}^{p}-\frac{1}{1-q} \int_{\Omega} f(x)|u|^{1-q} d x-\frac{1}{\gamma+1}\left[\|u\|_{\mu}^{p}-\int_{\Omega} f(x) u^{1-q} d x\right] \\
& =\left(\frac{1}{p}-\frac{1}{\gamma+1}\right)\|u\|_{\mu}^{p}-\left(\frac{1}{1-q}-\frac{1}{\gamma+1}\right) \int_{\Omega} f(x) u^{1-q} d x \\
& \geq\left(\frac{1}{p}-\frac{1}{\gamma+1}\right)\|u\|_{\mu}^{p}-\left(\frac{1}{1-q}-\frac{1}{\gamma+1}\right)\|f\|_{\infty} \frac{|\Omega|^{\frac{p^{*}-1+q}{p^{*}}}}{\left(\sqrt[p]{S_{\mu}}\right)^{1-q}}\|u\|_{\mu}^{1-q} \\
& :=\mathcal{K}\left(\|u\|_{\mu}\right) .
\end{aligned}
$$

Let

$$
\mathcal{K}^{\prime}\left(\|u\|_{\mu}\right)=\left(1-\frac{p}{\gamma+1}\right)\|u\|_{\mu}^{p-1}-\left(1-\frac{1-q}{\gamma+1}\right)\|f\|_{\infty} \frac{|\Omega|^{\frac{p^{*}-1+q}{p^{*}}}}{\left(\sqrt[p]{S_{\mu}}\right)^{1-q}}\|u\|_{\mu}^{-q}=0 .
$$

We have

$$
\|u\|_{\mu}:=\left(\|u\|_{\mu}\right)_{\min }=\left[\frac{\left(1-\frac{1-q}{\gamma+1}\right)\|f\|_{\infty} \frac{|\Omega| \frac{p^{*}-1+q}{p^{*}}}{\left(\sqrt[p]{S_{\mu}}\right)^{1-q}}}{1-\frac{p}{\gamma+1}}\right]^{\frac{1}{p+q-1}} .
$$

Since $\mathcal{K}^{\prime \prime}\left(\|u\|_{\mu}\right)>0$ for all $\|u\|_{\mu}>0$ with $\mathcal{K}\left(\|u\|_{\mu}\right) \rightarrow 0$ as $\|u\|_{\mu} \rightarrow 0$ and $\mathcal{K}\left(\|u\|_{\mu}\right) \rightarrow \infty$ as $\|u\|_{\mu} \rightarrow \infty$. Therefore $\mathcal{K}(u)$ attains its minimum at $\left(\|u\|_{\mu}\right)_{\min }$, and

$$
\begin{aligned}
\mathcal{K}\left(\left(\|u\|_{\mu}\right)_{\min }\right)= & \left(\frac{1}{p}-\frac{1}{\gamma+1}\right)\left[\frac{\left(1-\frac{1-q}{\gamma+1}\right)\|f\|_{\infty} \frac{|\Omega|^{\frac{p^{*}-1+q}{p^{*}}}}{\left(p \sqrt{S_{\mu}}\right)^{1-q}}}{1-\frac{p}{\gamma+1}}\right]^{\frac{p}{p+q-1}} \\
& -\left(\frac{1}{1-q}-\frac{1}{\gamma+1}\right)\|f\|_{\infty} \frac{|\Omega|^{\frac{p^{*}-1+q}{p^{*}}}}{\left(\sqrt[p]{S_{\mu}}\right)^{1-q}}\left[\frac{\left(1-\frac{1-q}{\gamma+1}\right)\|f\|_{\infty} \frac{|\Omega|^{\frac{p^{*}-1+q}{p^{*}}}}{\left(\sqrt[p]{S_{\mu}}\right)^{1-q}}}{1-\frac{p}{\gamma+1}}\right]^{\frac{1-q}{p+q-1}} .
\end{aligned}
$$

By (3.1), we deduce that

$$
\lim _{\|u\|_{\mu} \rightarrow \infty} I_{\lambda, \mu}(u) \geq \lim _{\|u\|_{\mu} \rightarrow \infty} \mathcal{K}\left(\|u\|_{\mu}\right)=\infty
$$

namely, $I_{\lambda, \mu}(u)$ is coercive on $\mathcal{M}$. Combining with (3.1), we have

$$
I_{\lambda, \mu}(u) \geq \mathcal{K}(u) \geq \mathcal{K}\left(\left(\|u\|_{\mu}\right)_{\min }\right) .
$$

Thus $I_{\lambda, \mu}(u)$ is bounded below on $\mathcal{M}$. According to Lemma 2.3, if $\lambda \in\left(0, T_{\mu}\right)$, then $\mathcal{M}^{+} \cup \mathcal{M}^{0}$ and $\mathcal{M}^{-}$are two closed sets in $\mathcal{M}$. Therefore, we apply the Ekeland variational 
principle [2] to derive a minimizing sequence $\left\{u_{n}\right\} \subset \mathcal{M}^{+} \cup \mathcal{M}^{0}$ satisfying:

(i) $I_{\lambda, \mu}\left(u_{n}\right)<\inf _{\mathcal{M}^{+} \cup \mathcal{M}^{0}} I_{\lambda, \mu}(u)+\frac{1}{n}$;

(ii) $\quad I_{\lambda, \mu}(u) \geq I_{\lambda, \mu}\left(u_{n}\right)-\frac{1}{n}\left\|u-u_{n}\right\|, \quad \forall u \in \mathcal{M}^{+} \cup \mathcal{M}^{0}$.

Assume that $u_{n} \geq 0$ on $\Omega \backslash\{0\}$. Note that $I_{\lambda, \mu}(u)$ is bounded below on $\mathcal{M}$. By (3.2), we get

$$
\mathcal{K}\left(\left(\left\|u_{n}\right\|_{\mu}\right)_{\min }\right) \leq I_{\lambda, \mu}\left(u_{n}\right)<\inf _{\mathcal{M}^{+} \cup \mathcal{M}^{0}} I_{\lambda, \mu}(u)+\frac{1}{n} \leq C_{1},
$$

for $n$ large enough and a positive constant $C_{1}$. Hence $\left\{u_{n}\right\}$ is bounded in $\mathcal{M}$. Let us, for a subsequence, suppose that

$$
\begin{cases}u_{n} \rightarrow u_{0} & \text { in } W, \\ u_{n}(x) \rightarrow u_{0}(x) & \text { a.e. in } \Omega, \\ u_{n} \rightarrow u_{0} & \text { in } L^{1-q}(\Omega) \text { and } L^{\gamma+1}(\Omega) .\end{cases}
$$

For every $u \in \mathcal{M}^{+}$, we deduce from $p>1$ that

$$
\begin{aligned}
I_{\lambda, \mu}(u) & =\frac{1}{p}\|u\|_{\mu}^{p}-\frac{1}{1-q} \int_{\Omega} f(x)|u|^{1-q} d x-\frac{\lambda}{\gamma+1} \int_{\Omega} g(x)|u|^{\gamma+1} d x \\
& =\frac{1}{p}\|u\|_{\mu}^{p}-\frac{1}{1-q}\left[\|u\|_{\mu}^{p}-\lambda \int_{\Omega} g(x)|u|^{\gamma+1} d x\right]-\frac{\lambda}{\gamma+1} \int_{\Omega} g(x)|u|^{\gamma+1} d x \\
& =\left(\frac{1}{p}-\frac{1}{1-q}\right)\|u\|_{\mu}^{p}+\left(\frac{1}{1-q}-\frac{1}{\gamma+1}\right) \lambda \int_{\Omega} g(x)|u|^{\gamma+1} d x \\
& <\left(\frac{1}{p}-\frac{1}{1-q}\right)\|u\|_{\mu}^{p}+\left(\frac{1}{1-q}-\frac{1}{\gamma+1}\right) \frac{p+q-1}{\gamma+q}\|u\|_{\mu}^{p} \\
& =\frac{p+q-1}{\gamma+q}\left(\frac{1}{\gamma+1}-\frac{1}{p}\right)\|u\|_{\mu}^{p}<0,
\end{aligned}
$$

which implies that $\inf _{\mathcal{M}^{+}} I_{\lambda, \mu}(u)<0$. For $\lambda \in\left(0, T_{\mu}\right)$, it follows from Lemma 2.1 that $\mathcal{M}^{0}=$ $\{0\}$. Thus $u_{n} \in \mathcal{M}^{+}$for $n$ large enough and $\inf _{\mathcal{M}^{+} \cup \mathcal{M}^{0}} I_{\lambda, \mu}(u)=\inf _{\mathcal{M}^{+}} I_{\lambda, \mu}(u)<0$. Therefore

$$
I_{\lambda, \mu}\left(u_{0}\right) \leq \liminf _{n \rightarrow \infty} I_{\lambda, \mu}\left(u_{n}\right)=\inf _{\mathcal{M}^{+} \cup \mathcal{M}^{0}} I_{\lambda, \mu}<0
$$

i.e., $u_{0} \geq 0$ and $u_{0} \neq 0$.

In the following, we prove that, when $\lambda \in\left(0, T_{\mu}\right)$,

$$
(p+q-1) \int_{\Omega} f(x) u_{0}^{1-q} d x>\lambda(\gamma-q+1) \int_{\Omega} g(x) u_{0}^{\gamma+1} d x
$$

For $\left\{u_{n}\right\} \subset \mathcal{M}^{+}$, we have

$$
(p+q-1) \int_{\Omega} f(x) u_{0}^{1-q} d x-\lambda(\gamma-p+1) \int_{\Omega} g(x) u_{0}^{\gamma+1} d x
$$




$$
\begin{aligned}
& =\lim _{n \rightarrow \infty}\left[(p+q-1) \int_{\Omega} f(x) u_{n}^{1-q} d x-\lambda(\gamma-p+1) \int_{\Omega} g(x) u_{n}^{\gamma+1} d x\right] \\
& =\lim _{n \rightarrow \infty}\left\{(p+q-1)\left[\left\|u_{n}\right\|_{\mu}^{p}-\lambda \int_{\Omega} g(x) u_{n}^{\gamma+1} d x\right]-\lambda(\gamma-p+1) \int_{\Omega} g(x) u_{n}^{\gamma+1} d x\right\} \\
& =\lim _{n \rightarrow \infty}\left[(p+q-1)\left\|u_{n}\right\|_{\mu}^{p}-\lambda(\gamma+q) \int_{\Omega} g(x) u_{n}^{\gamma+1} d x\right] \geq 0 .
\end{aligned}
$$

We suppose that

$$
(p+q-1) \int_{\Omega} f(x) u_{0}^{1-q} d x-\lambda(\gamma-p+1) \int_{\Omega} g(x) u_{0}^{\gamma+1} d x=0 .
$$

It follows from $u_{n} \in \mathcal{M}$, the weak lower semi-continuity of the norm and (3.5) that

$$
\begin{aligned}
0 & =\lim _{n \rightarrow \infty}\left[\left\|u_{n}\right\|_{\mu}^{p}-\int_{\Omega} f(x) u_{n}^{1-q} d x-\lambda \int_{\Omega} g(x) u_{n}^{\gamma+1} d x\right] \\
& \geq\left\|u_{0}\right\|_{\mu}^{p}-\int_{\Omega} f(x) u_{0}^{1-q} d x-\lambda \int_{\Omega} g(x) u_{0}^{\gamma+1} d x \\
& =\left\{\begin{array}{l}
\left\|u_{0}\right\|_{\mu}^{p}-\lambda \frac{\gamma+q}{p+q-1} \int_{\Omega} g(x) u_{0}^{\gamma+1} d x, \\
\left\|u_{0}\right\|_{\mu}^{p}-\lambda \frac{\gamma+q}{\gamma-p+1} \int_{\Omega} f(x) u_{0}^{1-q} d x .
\end{array}\right.
\end{aligned}
$$

Hence, for every $\lambda \in\left(0, T_{\mu}\right)$ and $u_{0} \neq 0$, combining with (2.2), we obtain

$$
\begin{aligned}
0 & <A(\mu, \lambda)\left\|u_{0}\right\|_{\mu}^{\gamma+1} \\
& \leq\left(\frac{q+p-1}{q+\gamma}\right)\left(\frac{\gamma-p+1}{q+\gamma}\right)^{\frac{p-\gamma-1}{1-q-p}} \frac{\left(\left\|u_{0}\right\|_{\mu}^{p}\right)^{\frac{-\gamma-q}{1-q-p}}}{\left(\int_{\Omega} f(x)\left|u_{0}\right|^{1-q} d x\right)^{\frac{p-\gamma-1}{1-q-p}}}-\lambda \int_{\Omega} g(x)\left|u_{0}\right|^{\gamma+1} d x \\
& \leq\left(\frac{q+p-1}{q+\gamma}\right)\left(\frac{\gamma-p+1}{q+\gamma}\right)^{\frac{p-\gamma-1}{1-q-p}} \frac{\left(\left\|u_{0}\right\|_{\mu}^{p}\right)^{\frac{-\gamma-q}{1-q-p}}}{\left(\frac{\gamma-p+1}{q+\gamma}\left\|u_{0}\right\|_{\mu}^{p}\right)^{\frac{p-\gamma-1}{1-q-p}}}-\frac{p+q-1}{\gamma+q}\left\|u_{0}\right\|_{\mu}^{p}=0,
\end{aligned}
$$

which is a contradiction. In view of (3.4), we get

$$
(p+q-1) \int_{\Omega} f(x) u_{n}^{1-q} d x-\lambda(\gamma-p+1) \int_{\Omega} g(x) u_{n}^{\gamma+1} d x \geq C_{2}
$$

for $n$ large enough and some positive constant $C_{2}$. Since $u_{n} \in \mathcal{M}$, we have

$$
(p+q-1)\left\|u_{n}\right\|_{\mu}^{p}-\lambda(\gamma+q) \int_{\Omega} g(x) u_{n}^{\gamma+1} d x \geq C_{2}>0
$$

Set $\phi \in \mathcal{M}$ with $\phi \geq 0$. Using Lemma 2.4 , there exists $\widetilde{g}_{n}(t)$ such that $\widetilde{g}_{n}(0)=1$ and $\widetilde{g}_{n}(t)\left(u_{n}+\right.$ $t \phi) \in \mathcal{M}^{+}$. Thus

$$
\left\|u_{n}\right\|_{\mu}^{p}-\int_{\Omega} f(x) u_{n}^{1-q} d x-\lambda \int_{\Omega} g(x) u_{n}^{\gamma+1} d x=0
$$

and

$$
\tilde{g}_{n}^{p}(t)\left\|u_{n}+t \phi\right\|_{\mu}^{p}-\widetilde{g}_{n}^{1-q}(t) \int_{\Omega} f(x)\left(u_{n}+t \phi\right)^{1-q} d x-\lambda \widetilde{g}_{n}^{\gamma+1}(t) \int_{\Omega} g(x)\left(u_{n}+t \phi\right)^{\gamma+1} d x=0 .
$$


Therefore

$$
\begin{aligned}
0= & {\left[\widetilde{g}_{n}^{p}(t)-1\right]\left\|u_{n}+t \phi\right\|_{\mu}^{p}+\left(\left\|u_{n}+t \phi\right\|_{\mu}^{p}-\left\|u_{n}\right\|_{\mu}^{p}\right) } \\
& -\left[\widetilde{g}_{n}^{1-q}(t)-1\right] \int_{\Omega} f(x)\left(u_{n}+t \phi\right)^{1-q} d x \\
& -\int_{\Omega} f(x)\left[\left(u_{n}+t \phi\right)^{1-q}-u_{n}^{1-q}\right] d x-\lambda\left[\widetilde{g}_{n}^{\gamma+1}(t)-1\right] \int_{\Omega} g(x)\left(u_{n}+t \phi\right)^{\gamma+1} d x \\
& -\lambda \int_{\Omega} g(x)\left[\left(u_{n}+t \phi\right)^{\gamma+1}-u_{n}^{\gamma+1}\right] d x \\
\leq & {\left[\widetilde{g}_{n}^{p}(t)-1\right]\left\|u_{n}+t \phi\right\|_{\mu}^{p}+\left(\left\|u_{n}+t \phi\right\|_{\mu}^{p}-\left\|u_{n}\right\|_{\mu}^{p}\right) } \\
& -\left[\widetilde{g}_{n}^{1-q}(t)-1\right] \int_{\Omega} f(x)\left(u_{n}+t \phi\right)^{1-q} d x \\
& -\lambda\left[\widetilde{g}_{n}^{\gamma+1}(t)-1\right] \int_{\Omega} g(x)\left(u_{n}+t \phi\right)^{\gamma+1} d x-\lambda \int_{\Omega} g(x)\left[\left(u_{n}+t \phi\right)^{\gamma+1}-u_{n}^{\gamma+1}\right] d x .
\end{aligned}
$$

Dividing by $t>0$ and letting $t \rightarrow 0$, we have

$$
\begin{aligned}
0 \leq & p \widetilde{g}_{n}^{\prime}(0)\left\|u_{n}\right\|_{\mu}^{p}+p \int_{\Omega}\left(\left|\Delta u_{n}\right|^{p-2} \Delta u_{n} \Delta \phi-\mu \frac{\left|u_{n}\right|^{p-2} u_{n} \phi}{|x|^{2 p}}\right) d x \\
& -(1-q) \widetilde{g}_{n}^{\prime}(0) \int_{\Omega} f(x) u_{n}^{1-q} d x \\
& -\lambda(\gamma+1) \tilde{g}_{n}^{\prime}(0) \int_{\Omega} g(x) u_{n}^{\gamma+1} d x-\lambda(\gamma+1) \int_{\Omega} g(x) u_{n}^{\gamma} \phi d x \\
= & \tilde{g}_{n}^{\prime}(0)\left[p\left\|u_{n}\right\|_{\mu}^{p}-(1-q) \int_{\Omega} g(x) u_{n}^{\gamma+1} d x\right] \\
& +p \int_{\Omega}\left(\left|\Delta u_{n}\right|^{p-2} \Delta u_{n} \Delta \phi-\mu \frac{\left|u_{n}\right|^{p-2} u_{n} \phi}{|x|^{2 p}}\right) d x \\
& -\lambda(\gamma+1) \int_{\Omega} g(x) u_{n}^{\gamma} \phi d x \\
= & \tilde{g}_{n}^{\prime}(0)\left[(p+q-1)\left\|u_{n}\right\|_{\mu}^{p}-\lambda(\gamma+q) \int_{\Omega} g(x) u_{n}^{\gamma+1} d x\right] \\
& +p \int_{\Omega}\left(\left|\Delta u_{n}\right|^{p-2} \Delta u_{n} \Delta \phi-\mu \frac{\left|u_{n}\right|^{p-2} u_{n} \phi}{|x|^{2 p}}\right) d x-\lambda(\gamma+1) \int_{\Omega} g(x) u_{n}^{\gamma} \phi d x,
\end{aligned}
$$

where $\widetilde{g}_{n}^{\prime}(0)$ denotes the right derivative of $\widetilde{g}_{n}(t)$ at zero. If it does not exist, $\widetilde{g}_{n}^{\prime}(0)$ should be replaced by $\lim _{k \rightarrow \infty} \frac{\widetilde{g}_{n}\left(t_{k}\right)-\widetilde{g}_{n}(0)}{t_{k}}$ for some sequence $\left\{t_{k}\right\}_{k=1}^{\infty}$ with $\lim _{k \rightarrow \infty} t_{k}=0$ and $t_{k}>0$.

Combining with (3.7) and (3.8), we have $\tilde{g}_{n}^{\prime}(0) \neq-\infty$. Now we prove that $\tilde{g}_{n}^{\prime}(0) \neq+\infty$. Otherwise, we suppose that $\widetilde{g}_{n}^{\prime}(0)=+\infty$. Note that $\widetilde{g}_{n}(t)>\widetilde{g}_{n}(0)=1$ for $n$ large enough, and

$$
\begin{aligned}
\left|\widetilde{g}_{n}(t)-1\right| \cdot\left\|u_{n}\right\|+t \widetilde{g}_{n}(t)\|\phi\| & \geq\left\|\left[\widetilde{g}_{n}(t)-1\right] u_{n}+t \widetilde{g}_{n}(t) \phi\right\| \\
& =\left\|\widetilde{g}_{n}(t)\left(u_{n}+t \phi\right)-u_{n}\right\| .
\end{aligned}
$$

Using condition (ii) with $u=\widetilde{g}_{n}(t)\left(u_{n}+t \phi\right) \in \mathcal{M}^{+}$, we deduce that

$$
\left[\widetilde{g}_{n}(t)-1\right] \cdot \frac{\left\|u_{n}\right\|}{n}+t \widetilde{g}_{n}(t) \frac{\|\phi\|}{n}
$$


Sang and Gro Journal of Inequalities and Applications

(2019) 2019:26

Page 17 of 26

$$
\begin{aligned}
& \geq \frac{1}{n}\left\|\widetilde{g}_{n}(t)\left(u_{n}+t \phi\right)-u_{n}\right\| \\
& \geq I_{\lambda, \mu}\left(u_{n}\right)-I_{\lambda, \mu}\left(\widetilde{g}_{n}(t)\left(u_{n}+t \phi\right)\right) \\
& =\frac{1}{p}\left\|u_{n}\right\|_{\mu}^{p}-\frac{1}{1-q} \int_{\Omega} f(x)\left|u_{n}\right|^{1-q} d x-\frac{\lambda}{\gamma+1} \int_{\Omega} g(x)\left|u_{n}\right|^{\gamma+1} d x-\frac{1}{p} \widetilde{g}_{n}^{p}(t)\left\|u_{n}+t \phi\right\|_{\mu}^{p} \\
& +\frac{1}{1-q} \int_{\Omega} f(x)\left|\widetilde{g}_{n}\left(u_{n}+t \phi\right)\right|^{1-q} d x+\frac{\lambda}{\gamma+1} \int_{\Omega} g(x)\left|\widetilde{g}_{n}\left(u_{n}+t \phi\right)\right|^{\gamma+1} d x \\
& =\frac{1}{p}\left\|u_{n}\right\|_{\mu}^{p}-\frac{1}{1-q}\left[\left\|u_{n}\right\|_{\mu}^{p}-\lambda \int_{\Omega} g(x)\left|u_{n}\right|^{\gamma+1} d x\right]-\frac{\lambda}{\gamma+1} \int_{\Omega} g(x)\left|u_{n}\right|^{\gamma+1} d x \\
& -\frac{1}{p} \widetilde{g}_{n}^{p}(t)\left\|u_{n}+t \phi\right\|_{\mu}^{p}+\frac{1}{1-q}\left[\widetilde{g}_{n}^{p}(t)\left\|u_{n}+t \phi\right\|_{\mu}^{p}-\lambda \int_{\Omega} g(x)\left|u_{n}+t \phi\right|^{\gamma+1} d x\right] \\
& +\frac{\lambda}{\gamma+1} \widetilde{g}_{n}^{\gamma+1}(t) \int_{\Omega} g(x)\left|u_{n}+t \phi\right|^{\gamma+1} d x \\
& =\left(\frac{1}{p}-\frac{1}{1-q}\right)\left\|u_{n}\right\|_{\mu}^{p}+\left(\frac{1}{1-q}-\frac{1}{\gamma+1}\right) \lambda \int_{\Omega} g(x)\left|u_{n}\right|^{\gamma+1} d x \\
& +\left(\frac{1}{1-q}-\frac{1}{p}\right) \widetilde{g}_{n}^{p}(t)\left\|u_{n}+t \phi\right\|_{\mu}^{p} \\
& -\left(\frac{1}{1-q}-\frac{1}{\gamma+1}\right) \lambda \widetilde{g}_{n}^{\gamma+1}(t) \int_{\Omega} g(x)\left|u_{n}+t \phi\right|^{\gamma+1} d x \\
& =\left(\frac{1}{1-q}-\frac{1}{p}\right)\left(\left\|u_{n}+t \phi\right\|_{\mu}^{p}-\left\|u_{n}\right\|_{\mu}^{p}\right)+\left(\frac{1}{1-q}-\frac{1}{p}\right)\left[\widetilde{g}_{n}^{p}(t)-1\right]\left\|u_{n}+t \phi\right\|_{\mu}^{p} \\
& -\left(\frac{1}{1-q}-\frac{1}{\gamma+1}\right) \lambda \widetilde{g}_{n}^{\gamma+1}(t) \int_{\Omega} g(x)\left[\left(u_{n}+t \phi\right)^{\gamma+1}-u_{n}^{\gamma+1}\right] d x \\
& -\left(\frac{1}{1-q}-\frac{1}{\gamma+1}\right) \lambda\left[\widetilde{g}_{n}^{\gamma+1}(t)-1\right] \int_{\Omega} g(x) u_{n}^{\gamma+1} d x \text {. }
\end{aligned}
$$

Dividing by $t>0$ and letting $t \rightarrow 0$, we obtain

$$
\begin{aligned}
\widetilde{g}_{n}^{\prime}(0) & \cdot \frac{\left\|u_{n}\right\|}{n}+\frac{\|\phi\|}{n} \\
\geq & \left(\frac{1}{1-q}-\frac{1}{p}\right) \cdot p \int_{\Omega}\left(\left|\Delta u_{n}\right|^{p-2} \Delta u_{n} \Delta \phi-\mu \frac{\left|u_{n}\right|^{p-2} u_{n} \phi}{|x|^{2 p}}\right) d x \\
& +\left(\frac{1}{1-q}-\frac{1}{p}\right) \cdot p \widetilde{g}_{n}^{\prime}(0)\left\|u_{n}\right\|_{\mu}^{p} \\
& -\lambda\left(\frac{1}{1-q}-\frac{1}{\gamma+1}\right)(\gamma+1) \int_{\Omega} g(x) u_{n}^{\gamma} \phi d x \\
& -\lambda\left(\frac{1}{1-q}-\frac{1}{\gamma+1}\right)(\gamma+1) \widetilde{g}_{n}^{\prime}(0) \int_{\Omega} g(x) u_{n}^{\gamma+1} d x \\
= & \frac{p}{1-q} \int_{\Omega}\left(\left|\Delta u_{n}\right|^{p-2} \Delta u_{n} \Delta \phi-\mu \frac{\left|u_{n}\right|^{p-2} u_{n} \phi}{|x|^{2 p}}\right) d x+\frac{p-1+q}{1-q} \widetilde{g}_{n}^{\prime}(0)\left\|u_{n}\right\|_{\mu}^{p} \\
& -\lambda \frac{\gamma+q}{1-q} \int_{\Omega} g(x) u_{n}^{\gamma} \phi d x-\lambda \frac{\gamma+q}{1-q} \widetilde{g}_{n}^{\prime}(0) \int_{\Omega} g(x) u_{n}^{\gamma+1} d x \\
= & \frac{\widetilde{g}_{n}^{\prime}(0)}{1-q}\left[(p-1+q)\left\|u_{n}\right\|_{\mu}^{p}-\lambda(\gamma+q) \int_{\Omega} g(x) u_{n}^{\gamma+1} d x\right] \\
& +\frac{p-1+q}{1-q} \int_{\Omega}\left(\left|\Delta u_{n}\right|^{p-2} \Delta u_{n} \Delta \phi-\mu \frac{\left|u_{n}\right|^{p-2} u_{n} \phi}{|x|^{2 p}}\right) d x-\lambda \frac{\gamma+q}{1-q} \int_{\Omega} g(x) u_{n}^{\gamma} \phi d x,
\end{aligned}
$$


that is,

$$
\begin{aligned}
\frac{\|\phi\|}{n} \geq & \frac{\tilde{g}_{n}^{\prime}(0)}{1-q}\left[(p-1+q)\left\|u_{n}\right\|_{\mu}^{p}-\lambda(\gamma+q) \int_{\Omega} g(x) u_{n}^{\gamma+1} d x-\frac{(1-q)\left\|u_{n}\right\|}{n}\right] \\
& +\frac{p-1+q}{1-q} \int_{\Omega}\left(\left|\Delta u_{n}\right|^{p-2} \Delta u_{n} \Delta \phi-\mu \frac{\left|u_{n}\right|^{p-2} u_{n} \phi}{|x|^{2 p}}\right) d x \\
& -\lambda \frac{\gamma+q}{1-q} \int_{\Omega} g(x) u_{n}^{\gamma} \phi d x,
\end{aligned}
$$

which is not true since $\widetilde{g}_{n}^{\prime}(0)=+\infty$ and

$$
(p-1+q)\left\|u_{n}\right\|_{\mu}^{p}-\lambda(\gamma+q) \int_{\Omega} g(x) u_{n}^{\gamma+1} d x-\frac{(1-q)\left\|u_{n}\right\|}{n} \geq C_{2}-\frac{(1-q) C_{3}}{n}>0 .
$$

It follows from (3.7), (3.8) and (3.10) that

$$
\left|\widetilde{g}_{n}^{\prime}(0)\right| \leq C_{4}
$$

for $n$ sufficiently large and a suitable positive constant $C_{4}$.

In the following, we prove that $u_{0} \in \mathcal{M}^{+}$is a solution of problem (1.1). By (3.9) and condition (ii) again, we have

$$
\begin{aligned}
& \frac{1}{n}\left[\left|\widetilde{g}_{n}(t)-1\right| \cdot\left\|u_{n}\right\|+t \widetilde{g}_{n}(t)\|\phi\|\right] \\
& \geq \\
& \geq \frac{1}{n}\left\|\widetilde{g}_{n}(t)\left(u_{n}+t \phi\right)-u_{n}\right\| \\
& \geq I_{\lambda, \mu}\left(u_{n}\right)-I_{\lambda, \mu}\left(\widetilde{g}_{n}(t)\left(u_{n}+t \phi\right)\right) \\
&= \frac{1}{p}\left\|u_{n}\right\|_{\mu}^{p}-\frac{1}{1-q} \int_{\Omega} f(x)\left|u_{n}\right|^{1-q} d x-\frac{\lambda}{\gamma+1} \int_{\Omega} g(x)\left|u_{n}\right|^{\gamma+1} d x-\frac{1}{p} \widetilde{g}_{n}^{p}(t)\left\|u_{n}+t \phi\right\|_{\mu}^{p} \\
&+\frac{1}{1-q} \int_{\Omega} f(x)\left|\widetilde{g}_{n}\left(u_{n}+t \phi\right)\right|^{1-q} d x+\frac{\lambda}{\gamma+1} \int_{\Omega} g(x)\left|\widetilde{g}_{n}\left(u_{n}+t \phi\right)\right|^{\gamma+1} d x \\
&=-\frac{\widetilde{g}_{n}^{p}(t)-1}{p}\left\|u_{n}\right\|_{\mu}^{p}-\frac{\widetilde{g}_{n}^{p}(t)}{p}\left(\left\|u_{n}+t \phi\right\|_{\mu}^{p}-\left\|u_{n}\right\|_{\mu}^{p}\right) \\
&+\frac{\widetilde{g}_{n}^{-q}(t)-1}{1-q} \int_{\Omega} f(x)\left(u_{n}+t \phi\right)^{1-q} d x \\
&+\frac{1}{1-q} \int_{\Omega} f(x)\left[\left(u_{n}+t \phi\right)^{1-q}-u_{n}^{1-q}\right] d x+\frac{\lambda\left(\widetilde{g}_{n}^{\gamma+1}(t)-1\right)}{\gamma+1} \int_{\Omega} g(x)\left(u_{n}+t \phi\right)^{\gamma+1} d x \\
&+\frac{\lambda}{\gamma+1} \int_{\Omega} g(x)\left[\left(u_{n}+t \phi\right)^{\gamma+1}-u_{n}^{\gamma+1}\right] d x .
\end{aligned}
$$

Dividing by $t>0$ and letting $t \rightarrow 0^{+}$, we derive that

$$
\begin{aligned}
& \frac{1}{n}\left[\left|\widetilde{g}_{n}^{\prime}(0)\right| \cdot\left\|u_{n}\right\|+\|\phi\|\right] \\
& \geq-\widetilde{g}_{n}^{\prime}(0)\left\|u_{n}\right\|_{\mu}^{p}-\int_{\Omega}\left(\left|\Delta u_{n}\right|^{p-2} \Delta u_{n} \Delta \phi-\mu \frac{\left|u_{n}\right|^{p-2} u_{n} \phi}{|x|^{2 p}}\right) d x+\widetilde{g}_{n}^{\prime}(0) \int_{\Omega} f(x) u_{n}^{1-q} d x \\
& \quad+\lambda \widetilde{g}_{n}^{\prime}(0) \int_{\Omega} g(x) u_{n}^{\gamma+1} d x+\lambda \int_{\Omega} g(x) u_{n}^{\gamma} \phi d x
\end{aligned}
$$




$$
\begin{aligned}
& +\liminf _{t \rightarrow 0^{+}} \frac{1}{1-q} \int_{\Omega} \frac{f(x)\left[\left(u_{n}+t \phi\right)^{1-q}-u_{n}^{1-q}\right]}{t} d x \\
= & -\widetilde{g}_{n}^{\prime}(0)\left[\left\|u_{n}\right\|_{\mu}^{p}-\int_{\Omega} f(x) u_{n}^{1-q} d x-\lambda \int_{\Omega} g(x) u_{n}^{\gamma+1} d x\right] \\
& -\int_{\Omega}\left(\left|\Delta u_{n}\right|^{p-2} \Delta u_{n} \Delta \phi-\mu \frac{\left|u_{n}\right|^{p-2} u_{n} \phi}{|x|^{2 p}}\right) d x+\lambda \int_{\Omega} g(x) u_{n}^{\gamma} \phi d x \\
& +\liminf _{t \rightarrow 0^{+}} \frac{1}{1-q} \int_{\Omega} \frac{f(x)\left[\left(u_{n}+t \phi\right)^{1-q}-u_{n}^{1-q}\right]}{t} d x .
\end{aligned}
$$

Noting $f(x)\left[\left(u_{n}+t \phi\right)^{1-q}-u_{n}^{1-q}\right] \geq 0$, for every $x \in \Omega$ and $t>0$, together with the Fatou lemma, we find that

$$
\liminf _{t \rightarrow 0^{+}}\left[\frac{f(x)\left[\left(u_{n}+t \phi\right)^{1-q}-u_{n}^{1-q}\right]}{t}\right]
$$

is integrable, and

$$
\begin{aligned}
& \int_{\Omega} f(x) u_{n}^{-q} \phi d x \\
& \quad \leq \liminf _{t \rightarrow 0^{+}} \frac{1}{1-q} \int_{\Omega} \frac{f(x)\left[\left(u_{n}+t \phi\right)^{1-q}-u_{n}^{1-q}\right]}{t} d x \\
& \leq \frac{\left|\widetilde{g}_{n}^{\prime}(0)\right|\left\|u_{n}\right\|+\|\phi\|}{n}+\int_{\Omega}\left(\left|\Delta u_{n}\right|^{p-2} \Delta u_{n} \Delta \phi-\mu \frac{\left|u_{n}\right|^{p-2} u_{n} \phi}{|x|^{2 p}}\right) d x \\
& \quad-\lambda \int_{\Omega} g(x) u_{n}^{\gamma} \phi d x \\
& \leq \frac{C_{3} C_{4}+\|\phi\|}{n}+\int_{\Omega}\left(\left|\Delta u_{n}\right|^{p-2} \Delta u_{n} \Delta \phi-\mu \frac{\left|u_{n}\right|^{p-2} u_{n} \phi}{|x|^{2 p}}\right) d x-\lambda \int_{\Omega} g(x) u_{n}^{\gamma} \phi d x .
\end{aligned}
$$

Applying the Fatou lemma again, we have

$$
\begin{aligned}
& \int_{\Omega} f(x) u_{0}^{-q} \phi d x \\
& =\int_{\Omega}\left[\liminf _{n \rightarrow \infty} f(x) u_{n}^{-q} \phi\right] d x \leq \liminf _{n \rightarrow \infty} \int_{\Omega} f(x) u_{n}^{-q} \phi d x \\
& \leq \liminf _{n \rightarrow \infty}\left[\frac{C_{3} C_{4}+\|\phi\|}{n}+\int_{\Omega}\left(\left|\Delta u_{n}\right|^{p-2} \Delta u_{n} \Delta \phi-\mu \frac{\left|u_{n}\right|^{p-2} u_{n} \phi}{|x|^{2 p}}\right) d x\right. \\
& \left.\quad-\lambda \int_{\Omega} g(x) u_{n}^{\gamma} \phi d x\right] \\
& =\int_{\Omega}\left(\left|\Delta u_{0}\right|^{p-2} \Delta u_{0} \Delta \phi-\mu \frac{\left|u_{0}\right|^{p-2} u_{0} \phi}{|x|^{2 p}}\right) d x-\lambda \int_{\Omega} g(x) u_{0}^{\gamma} \phi d x .
\end{aligned}
$$

Since $\int_{\Omega} u_{0}^{-q} \varphi_{1} d x<\infty$, we have $u_{0}>0$ a.e. in $\Omega$. For every $\phi \in \mathcal{M}$ and $\phi \geq 0$, we have

$$
\begin{aligned}
& \int_{\Omega}\left(\left|\Delta u_{0}\right|^{p-2} \Delta u_{0} \Delta \phi-\mu \frac{\left|u_{0}\right|^{p-2} u_{0} \phi}{|x|^{2 p}}\right) d x-\int_{\Omega} f(x) u_{0}^{-q} \phi d x \\
& -\lambda \int_{\Omega} g(x) u_{0}^{\gamma} \phi d x \geq 0 .
\end{aligned}
$$


Set $\phi=u_{0}$ in (3.11), we derive that

$$
\left\|u_{0}\right\|_{\mu}^{p}=\int_{\Omega}\left(\left|\Delta u_{0}\right|^{p}-\mu \frac{\left|u_{0}\right|^{p}}{|x|^{2 p}}\right) d x \geq \int_{\Omega} f(x) u_{0}^{1-q} d x+\lambda \int_{\Omega} g(x) u_{0}^{\gamma+1} d x .
$$

Furthermore

$$
\begin{aligned}
\left\|u_{0}\right\|_{\mu}^{p} & \leq \liminf _{n \rightarrow \infty}\left\|u_{n}\right\|_{\mu}^{p} \leq \limsup _{n \rightarrow \infty}\left\|u_{n}\right\|_{\mu}^{p} \\
& =\limsup _{n \rightarrow \infty}\left[\int_{\Omega} f(x) u_{n}^{1-q} d x+\lambda \int_{\Omega} g(x) u_{n}^{\gamma+1} d x\right] \\
& =\int_{\Omega} f(x) u_{0}^{1-q} d x+\lambda \int_{\Omega} g(x) u_{0}^{\gamma+1} d x
\end{aligned}
$$

Hence

$$
\left\|u_{0}\right\|_{\mu}^{p}=\int_{\Omega} f(x) u_{0}^{1-q} d x+\lambda \int_{\Omega} g(x) u_{0}^{\gamma+1} d x
$$

Therefore $u_{n} \rightarrow u_{0}$ in $\mathcal{M}$ and $u_{0} \in \mathcal{M}$. By (3.4), we have

$$
\begin{aligned}
(p & +q-1)\left\|u_{0}\right\|_{\mu}^{p}-\lambda(\gamma+q) \int_{\Omega} g(x) u_{0}^{\gamma+1} d x \\
& =(p+q-1)\left[\int_{\Omega} f(x) u_{0}^{1-q} d x+\lambda \int_{\Omega} g(x) u_{0}^{\gamma+1} d x\right]-\lambda(\gamma+q) \int_{\Omega} g(x) u_{0}^{\gamma+1} d x \\
& =(p+q-1) \int_{\Omega} f(x) u_{0}^{1-q} d x-\lambda(\gamma-1) \int_{\Omega} g(x) u_{0}^{\gamma+1} d x>0,
\end{aligned}
$$

i.e., $u_{0} \in \mathcal{M}^{+}$.

Next, we only need to show that $u_{0}$ is a positive weak solution of problem (1.1). Define

$$
\Phi=\left(u_{0}+\varepsilon \phi\right)^{+}, \quad \phi \in W, \varepsilon>0 .
$$

Substituting $\Phi$ into (3.11), combining with (3.12), we deduce that

$$
\begin{aligned}
0 \leq & \int_{\Omega}\left[\left|\Delta u_{0}\right|^{p-2} \Delta u_{0} \Delta \Phi-\mu \frac{\left|u_{0}\right|^{p-2} u_{0} \Phi}{|x|^{2 p}}-f(x) u_{0}^{-q} \Phi-\lambda g(x) u_{0}^{\gamma} \Phi\right] d x \\
= & \int_{\Omega_{1}}\left[\left|\Delta u_{0}\right|^{p-2} \Delta u_{0} \Delta \Phi-\mu \frac{\left|u_{0}\right|^{p-2} u_{0} \Phi}{|x|^{2 p}}-f(x) u_{0}^{-q} \Phi-\lambda g(x) u_{0}^{\gamma} \Phi\right] d x \\
& +\int_{\Omega_{2}}\left[\left|\Delta u_{0}\right|^{p-2} \Delta u_{0} \Delta \Phi-\mu \frac{\left|u_{0}\right|^{p-2} u_{0} \Phi}{|x|^{2 p}}-f(x) u_{0}^{-q} \Phi-\lambda g(x) u_{0}^{\gamma} \Phi\right] d x \\
= & \int_{\Omega}\left[\left|\Delta u_{0}\right|^{p-2} \Delta u_{0} \Delta\left(u_{0}+\varepsilon \phi\right)-\mu \frac{\left|u_{0}\right|^{p-2} u_{0}\left(u_{0}+\varepsilon \phi\right)}{|x|^{2 p}}-f(x) u_{0}^{-q}\left(u_{0}+\varepsilon \phi\right)\right. \\
& \left.-\lambda g(x) u_{0}^{\gamma}\left(u_{0}+\varepsilon \phi\right)\right] d x \\
& -\int_{\Omega_{2}}\left[\left|\Delta u_{0}\right|^{p-2} \Delta u_{0} \Delta\left(u_{0}+\varepsilon \phi\right)-\mu \frac{\left|u_{0}\right|^{p-2} u_{0}\left(u_{0}+\varepsilon \phi\right)}{|x|^{2 p}}-f(x) u_{0}^{-q}\left(u_{0}+\varepsilon \phi\right)\right. \\
& \left.-\lambda g(x) u_{0}^{\gamma}\left(u_{0}+\varepsilon \phi\right)\right] d x
\end{aligned}
$$




$$
\begin{aligned}
= & \int_{\Omega}\left[\left|\Delta u_{0}\right|^{p}-\mu \frac{\left|u_{0}\right|^{p}}{|x|^{2 p}}-f(x) u_{0}^{1-q}-\lambda g(x) u_{0}^{\gamma+1}\right] d x \\
& +\varepsilon \int_{\Omega}\left[\left|\Delta u_{0}\right|^{p-2} \Delta u_{0} \Delta \phi-\mu \frac{\left|u_{0}\right|^{p-2} u_{0} \phi}{|x|^{2 p}}-f(x) u_{0}^{-q} \phi-\lambda g(x) u_{0}^{\gamma} \phi\right] d x \\
& -\int_{\Omega_{2}}\left[\left|\Delta u_{0}\right|^{p}+\varepsilon\left|\Delta u_{0}\right|^{p-2} \Delta u_{0} \Delta \phi-\mu \frac{\left|u_{0}\right|^{p-2} u_{0}\left(u_{0}+\varepsilon \phi\right)}{|x|^{2 p}}\right] d x \\
& -\int_{\Omega_{2}}\left[-f(x) u_{0}^{-q}\left(u_{0}+\varepsilon \phi\right)-\lambda g(x) u_{0}^{\gamma+1}-\varepsilon \lambda g(x) u_{0}^{\gamma} \phi\right] d x \\
\leq & \varepsilon \int_{\Omega}\left[\left|\Delta u_{0}\right|^{p-2} \Delta u_{0} \Delta \phi-\mu \frac{\left|u_{0}\right|^{p-2} u_{0} \phi}{|x|^{2 p}}-f(x) u_{0}^{-q} \phi-\lambda g(x) u_{0}^{\gamma} \phi\right] d x \\
& -\varepsilon \int_{\Omega_{2}}\left|\Delta u_{0}\right|^{p-2} \Delta u_{0} \Delta \phi d x+\lambda\|g\|_{\infty} \int_{\Omega_{2}}|\varepsilon \phi|^{\gamma+1} d x+\varepsilon \lambda \int_{\Omega_{2}} g(x) u_{0}^{\gamma} \phi d x \\
= & \varepsilon \int_{\Omega}\left[\left|\Delta u_{0}\right|^{p-2} \Delta u_{0} \Delta \phi-\mu \frac{\left|u_{0}\right|^{p-2} u_{0} \phi}{|x|^{2 p}}-f(x) u_{0}^{-q} \phi-\lambda g(x) u_{0}^{\gamma} \phi\right] d x \\
& -\varepsilon \int_{\Omega_{2}}\left|\Delta u_{0}\right|^{p-2} \Delta u_{0} \Delta \phi d x+\varepsilon \lambda \varepsilon^{\gamma}\|g\|_{\infty} \int_{\Omega_{2}}|\phi|^{\gamma+1} d x+\varepsilon \lambda \int_{\Omega_{2}} g(x) u_{0}^{\gamma} \phi d x,
\end{aligned}
$$

where $\Omega_{1}=\left\{x \mid u_{0}(x)+\varepsilon \phi(x)>0, x \in \Omega\right\}$ and $\Omega_{2}=\left\{x \mid u_{0}(x)+\varepsilon \phi(x) \leq 0, x \in \Omega\right\}$. Since the measure of $\Omega_{2}$ tends to zero as $\varepsilon \rightarrow 0$, we have $\int_{\Omega_{2}}\left|\Delta u_{0}\right|^{p-2} \Delta u_{0} \Delta \phi d x \rightarrow 0$ as $\varepsilon \rightarrow 0$. By the same arguments, we have $\lambda \varepsilon^{\gamma}\|g\|_{\infty} \int_{\Omega_{2}}|\phi|^{\gamma+1} d x \longrightarrow 0$ and $\lambda \int_{\Omega_{2}} g(x) u_{0}^{\gamma} \phi d x \longrightarrow 0$ as $\varepsilon \rightarrow 0$. Dividing by $\varepsilon$ and taking the limit for $\varepsilon \rightarrow 0$, we deduce that

$$
\int_{\Omega}\left[\left|\Delta u_{0}\right|^{p-2} \Delta u_{0} \Delta \phi-\mu \frac{\left|u_{0}\right|^{p-2} u_{0} \phi}{|x|^{2 p}}-f(x) u_{0}^{-q} \phi-\lambda g(x) u_{0}^{\gamma} \phi\right] d x \geq 0 .
$$

Therefore $u_{0}$ is a positive weak solution of problem (1.1).

We adopt the Ekeland variational principle again to derive a minimizing sequence $U_{n} \subset$ $\mathcal{M}^{-}$for the minimization problem $\inf _{\mathcal{M}} I_{\lambda, \mu}$ such that for $U_{n} \in \mathcal{M}, U_{n} \rightarrow U_{0}$ weakly in $\mathcal{M}$ and pointwise a.e. in $\Omega$. By similar arguments to those in (3.4) and (3.6), for $\lambda \in\left(0, T_{\mu}\right)$, we have

$$
(p+q-1) \int_{\Omega} f(x)\left|U_{0}\right|^{1-q} d x-\lambda(\gamma-p+1) \int_{\Omega} g(x)\left|U_{0}\right|^{\gamma+1} d x<0
$$

which leads to

$$
(p+q-1) \int_{\Omega} f(x)\left|U_{n}\right|^{1-q} d x-\lambda(\gamma-p+1) \int_{\Omega} g(x)\left|U_{n}\right|^{\gamma+1} d x \leq-C_{5},
$$

for $n$ large enough and a positive constant $C_{5}$. Therefore $U_{0}>0$ is the positive weak solution of problem (1.1). Furthermore $U_{0} \in \mathcal{M}$. By (3.14), we obtain

$$
\begin{aligned}
(p & +q-1)\left\|U_{0}\right\|_{\mu}^{p}-(q+\gamma) \lambda \int_{\Omega} g(x) U_{0}^{\gamma+1} d x \\
& =(p+q-1)\left[\int_{\Omega} f(x) U_{0}^{1-q} d x+\lambda \int_{\Omega} g(x) U_{0}^{\gamma+1} d x\right]-\lambda(\gamma+q) \int_{\Omega} g(x) U_{0}^{\gamma+1} d x \\
& =(p+q-1) \int_{\Omega} f(x) U_{0}^{1-q} d x-\lambda(\gamma-p+1) \int_{\Omega} g(x) U_{0}^{\gamma+1} d x<0,
\end{aligned}
$$


i.e., $U_{0} \in \mathcal{M}^{-}$. According to Lemma 2.2, we know that problem (1.1) has at least two positive weak solutions $u_{0} \in \mathcal{M}^{+}$and $U_{0} \in \mathcal{M}^{-}$with $\left\|U_{0}\right\|_{\mu}>\left\|u_{0}\right\|_{\mu}$ for every $\lambda \in\left(0, T_{\mu}\right)$. This completes the proof of Theorem 1.1.

\section{Proof of Corollary 1.2}

For every $U \in \mathcal{M}^{-}$, by Lemma 2.2, we deduce that

$$
\begin{aligned}
\|U\|_{\mu} & >M_{\mu}(\lambda) \\
& =\left[\frac{p+q-1}{\lambda(\gamma+q)} \frac{1}{\|g\|_{\infty}} \frac{\left(\sqrt[p]{S_{\mu}}\right)^{\gamma+1}}{|\Omega|^{\frac{p^{*}-1-\gamma}{p^{*}}}}\right]^{\frac{1}{\gamma+1-p}} \\
& =\left(\frac{1}{\lambda}\right)^{\frac{1}{\gamma+1-p}}\left(\frac{p+q-1}{\gamma+q}\right)^{\frac{1}{\gamma+1-p}}\left(\frac{1}{\|g\|_{\infty}}\right)^{\frac{1}{\gamma+1-p}} \frac{\left(\sqrt[p]{S_{\mu}}\right)^{\frac{\gamma+1}{\gamma+1-p}}}{|\Omega|^{\frac{p^{*}-1-\gamma}{p^{*}(1-p)}}} \\
& =\left(T_{\mu}\right)^{-\frac{1}{\gamma+1-p}}\left(\frac{p+q-1}{\gamma+q}\right)^{\frac{1}{\gamma+1-p}}\left(\frac{1}{\|g\|_{\infty}}\right)^{\frac{1}{\gamma+1-p}} \frac{\left(\sqrt[p]{S_{\mu}}\right)^{\frac{\gamma+1}{\gamma+1-p}}}{|\Omega|^{\frac{p^{*}-1-\gamma}{p^{*}(\gamma+1-p)}}}\left(\frac{T_{\mu}}{\lambda}\right)^{\frac{1}{\gamma+1-p}} .
\end{aligned}
$$

Combining with the definition of $T_{\mu}$, we have

$$
\begin{aligned}
\|U\|_{\mu}> & \left(\frac{q+\gamma}{q+p-1}\right)^{\frac{1}{\gamma+1-p}}\left(\frac{q+\gamma}{\gamma-p+1}\right)^{\frac{1}{p+q-1}}\left(\|f\|_{\infty}\right)^{\frac{1}{p+q-1}}\left(\|g\|_{\infty}\right)^{\gamma-p+1} \frac{|\Omega|^{\frac{2 p}{N} \frac{q+\gamma}{p+q-1} \frac{1}{\gamma+1-p}}}{S_{\mu}^{\frac{q+\gamma}{p+q} \frac{1}{\gamma+1-p}}} \\
& \times\left(\frac{p+q-1}{\gamma+q}\right)^{\frac{1}{\gamma+1-p}}\left(\frac{1}{\|g\|_{\infty}}\right)^{\frac{1}{\gamma+1-p}} \frac{\left(\sqrt[p]{S_{\mu}}\right)^{\frac{\gamma+1}{\gamma+1-p}}}{|\Omega|^{\frac{p^{*}-1-\gamma}{p^{*}(\gamma+1-p)}}}\left(\frac{T_{\mu}}{\lambda}\right)^{\frac{1}{\gamma+1-p}} \\
= & \left(\frac{q+\gamma}{\gamma-p+1}\right)^{\frac{1}{p+q-1}}\left(\|f\|_{\infty}\right)^{\frac{1}{p+q-1}}\left(\frac{|\Omega|^{\frac{2 p}{N} \frac{q+\gamma}{p+q-1} \frac{1}{\gamma+1-p}-\frac{p^{*}-1-\gamma}{p^{*}(\gamma+1-p)}}}{\left(\sqrt[p]{S_{\mu}}\right)^{p \frac{q+\gamma}{p+q-1} \frac{1}{\gamma+1-p}-\frac{\gamma+1}{\gamma+1-p}}}\right)\left(\frac{T_{\mu}}{\lambda}\right)^{\frac{1}{\gamma+1-p}} \\
= & |\Omega|^{\frac{1}{p}}\left(\frac{q+\gamma}{\gamma-p+1}\right)^{\frac{1}{p+q-1}}\left(\|f\|_{\infty}\right)^{\frac{1}{p+q-1}}\left(\frac{|\Omega|^{\frac{2}{N}}}{\sqrt[p]{S_{\mu}}}\right)^{\frac{1-q}{p+q-1}}\left(\frac{T_{\mu}}{\lambda}\right)^{\frac{1}{\gamma+1-p}} \\
= & |\Omega|^{\frac{1}{p}}\left(\|f\|_{\infty}\right)^{\frac{1}{p+q-1}}\left(1+\frac{p+q-1}{\gamma-p+1}\right)^{\frac{1}{p+q-1}}\left(\frac{|\Omega|^{\frac{2}{N}}}{\sqrt[p]{S_{\mu}}}\right)^{\frac{1-q}{p+q-1}}\left(\frac{T_{\mu}}{\lambda}\right)^{\frac{1}{\gamma+1-p}}
\end{aligned}
$$

where we adopted the following facts:

$$
\begin{aligned}
& \frac{2 p}{N} \frac{q+\gamma}{p+q-1} \frac{1}{\gamma+1-p}-\frac{p^{*}-1-\gamma}{p^{*}(\gamma+1-p)} \\
& \quad=\frac{p^{*}-1+q}{p^{*}(p+q-1)}=\frac{\frac{N p}{N-2 p}+q-1}{\frac{N p}{N-2 p}(p+q-1)} \\
& \quad=\frac{N(p+q-1)+2 p(1-q)}{N p(p+q-1)}=\frac{1}{p}+\frac{2}{N} \cdot \frac{1-q}{p+q-1}, \\
& p \cdot \frac{q+\gamma}{p+q-1} \frac{1}{\gamma+1-p}-\frac{\gamma+1}{\gamma+1-p}=\frac{(1-q)(\gamma+1-p)}{(p+q-1)(\gamma+1-p)}=\frac{1-q}{p+q-1} .
\end{aligned}
$$


Let $U_{\lambda, \mu, \varepsilon} \in \mathcal{M}^{-}$be the solution of problem (1.1) with $\gamma=\varepsilon+p-1$, where $\lambda \in\left(0, T_{\mu}\right)$. Then

$$
\left\|U_{\lambda, \mu, \varepsilon}\right\|_{\mu}>C_{\mu, \varepsilon}\left(\frac{T_{\mu}}{\lambda}\right)^{\frac{1}{\varepsilon}}
$$

where $C_{\mu, \varepsilon}$ is given in (1.16). This completes the proof of Corollary 1.2.

\section{Proof of Theorem 1.3}

For simplicity, we consider problem (1.1) with $f=g=1$,

$$
\begin{cases}\Delta_{p}^{2} u-\mu \frac{|u|^{p-2} u}{|x|^{2 p}}=u^{-q}+\lambda u^{\gamma} & \text { in } \Omega \backslash\{0\}, \\ u(x)>0 & \text { in } \Omega \backslash\{0\}, \\ u=\Delta u=0 & \text { on } \partial \Omega .\end{cases}
$$

Let us define

$$
\lambda^{*}=\lambda^{*}(N, \Omega, \mu, q, \gamma)=\sup \{\lambda>0: \text { problem (5.1) has a positive solution }\} .
$$

Using Theorem 1.1, we provide uniform estimates for $\lambda^{*}(N, \Omega, \mu, q, \gamma)$.

Lemma 5.1 For $1<p<\frac{N}{2}, 0<\mu<\mu_{N, p}, 0<q<1<\gamma<p^{*}-1$ and $\Omega \in \mathbb{U}$, where $\mathbb{U}=\{\Omega \in$ $\mathbb{R}^{N}: \Omega$ is an open and bounded domain $\}$, we have

$$
0<\lambda^{-} \leq \lambda^{*} \leq \lambda^{+}<\infty
$$

where

$$
\lambda^{-}=\left(\frac{q+p-1}{q+\gamma}\right)\left(\frac{\gamma-p+1}{q+\gamma}\right)^{\frac{p-\gamma-1}{1-q-p}}\left[\frac{S_{\mu}}{|\Omega|^{\frac{2 p}{N}}}\right]^{\frac{q+\gamma}{p+q-1}}
$$

and

$$
\lambda^{+}=\lambda_{1}^{\frac{\gamma+q}{q-1+p}}\left(\frac{\gamma-p+1}{\gamma+q}\right)^{\frac{\gamma-p+1}{q+p-1}} \frac{-1+p+q}{\gamma+q}+\frac{1}{2} .
$$

Proof (1) Assume that $\lambda \in\left(0, \lambda^{-}\right)$, then problem (5.1) has at least two solutions. By the definition of $\lambda^{*}$, we have $\lambda^{*} \geq \lambda^{-}>0$.

(2) Assume that (5.1) has a positive solution $u$. Integrating over $\Omega$ by multiplying (5.1) by $\varphi_{1}$, we obtain

$$
\lambda_{1} \int_{\Omega}|u|^{p-2} u \varphi_{1} d x=\int_{\Omega}\left(\Delta_{p}^{2} u-\mu \frac{|u|^{p-2} u}{|x|^{2 p}}\right) \varphi_{1} d x=\int_{\Omega} u^{-q} \varphi_{1} d x+\lambda \int_{\Omega} u^{\gamma} \varphi_{1} d x .
$$

We claim that there exists $\lambda^{+}>0$ such that

$$
t^{-q}+\lambda^{+} t^{\gamma}>\lambda_{1} t^{p-1}, \quad \forall t>0 .
$$


In fact, letting

$$
F_{\lambda}(t)=t^{-q}+\lambda t^{\gamma}-\lambda_{1} t^{p-1}=t^{\gamma}\left(t^{-q-\gamma}+\lambda-\lambda_{1} t^{-\gamma+p-1}\right):=t^{\gamma} \cdot G_{\lambda}(t), \quad t>0 .
$$

We have

$$
G_{\lambda}^{\prime}(t)=(-\gamma-q) t^{-\gamma-q-1}+\lambda_{1}(\gamma-p+1) t^{-\gamma+p-2}=0
$$

i.e.,

$$
t:=t_{\min }=\left(\frac{\gamma+q}{\lambda_{1}(\gamma-p+1)}\right)^{\frac{1}{q-1+p}} .
$$

Then $G_{\lambda}(t)$ attains minimum at $t_{\mathrm{min}}$, and

$$
G_{\lambda}\left(t_{\min }\right)=\lambda+\lambda_{1}^{\frac{\gamma+q}{q-1+p}}\left(\frac{\gamma-p+1}{\gamma+q}\right)^{\frac{\gamma-p+1}{q+p-1}} \frac{1-p-q}{\gamma+q} .
$$

We may choose $\lambda=\lambda_{1}^{\frac{\gamma+q}{q-1+p}}\left(\frac{\gamma-p+1}{\gamma+q}\right)^{\frac{\gamma-p+1}{q+p-1}} \frac{-1+p+q}{\gamma+q}+\frac{1}{2}=\lambda^{+}>0$ such that

$$
G_{\lambda^{+}}(t) \geq G_{\lambda^{+}}\left(t_{\min }\right)=\frac{1}{2}>0, \quad \text { for } t>0 \text {. }
$$

Therefore

$$
F_{\lambda^{+}}(t)=t^{\gamma} \cdot G_{\lambda^{+}}(t)>0 \quad \text { for } t>0
$$

Using (5.3) with $t=u$, we have

$$
\int_{\Omega} u^{-q} \varphi_{1} d x+\lambda^{+} \int_{\Omega} u^{\gamma} \varphi_{1} d x \geq \lambda_{1} \int_{\Omega}|u|^{p-2} u \varphi_{1} d x
$$

Combining with (5.2) and (5.5), we obtain $\lambda \leq \lambda^{+}$. Since $\lambda$ is arbitrary, we have $\lambda^{*} \leq$ $\lambda^{+}<\infty$.

Proof of Theorem 1.3 We only prove the case that $0<\lambda<\lambda^{*}$. By the definition of $\lambda^{*}$, there exists $\bar{\lambda} \in\left(\lambda, \lambda^{*}\right)$ such that the problem

$$
\Delta_{p}^{2} u-\mu \frac{|u|^{p-2} u}{|x|^{2 p}}=u^{-q}+\bar{\lambda} u^{\gamma}
$$

has a positive solution, denoted by $u_{\bar{\lambda}}$. It follows that

$$
\Delta_{p}^{2} u_{\bar{\lambda}}-\mu \frac{\left|u_{\bar{\lambda}}\right|^{p-2} u_{\bar{\lambda}}}{|x|^{2 p}}=u_{\bar{\lambda}}^{-q}+\bar{\lambda} u_{\bar{\lambda}}^{\gamma} \geq u_{\bar{\lambda}}^{-q}+\lambda u_{\bar{\lambda}}^{\gamma} .
$$

Hence $u_{\bar{\lambda}}$ is an upper solution of (5.1). Note that $\lim _{t \rightarrow 0^{+}} G_{\lambda}(t)=\infty$, we can take $\varepsilon>0$ small enough with $\varepsilon \varphi_{1}<u_{\bar{\lambda}}$ and $G_{\lambda}\left(\varepsilon \varphi_{1}\right) \geq 0$. Thus

$$
F_{\lambda}\left(\varepsilon \varphi_{1}\right)=\left(\varepsilon \varphi_{1}\right)^{\gamma} G_{\lambda}\left(\varepsilon \varphi_{1}\right) \geq 0, \quad \text { for all } \lambda>0,
$$


i.e.,

$$
\lambda_{1}\left(\varepsilon \varphi_{1}\right)^{p-1} \leq\left(\varepsilon \varphi_{1}\right)^{-q}+\lambda\left(\varepsilon \varphi_{1}\right)^{\gamma}, \quad \text { for all } \lambda>0
$$

Combining with (1.10) and (5.6), we obtain

$$
\begin{aligned}
\Delta_{p}^{2}\left(\varepsilon \varphi_{1}\right)-\mu \frac{\left|\left(\varepsilon \varphi_{1}\right)\right|^{p-2}\left(\varepsilon \varphi_{1}\right)}{|x|^{2 p}} & =\varepsilon^{p-1}\left(\Delta_{p}^{2} \varphi_{1}-\mu \frac{\left|\varphi_{1}\right|^{p-2} \varphi_{1}}{|x|^{2 p}}\right) \\
& =\varepsilon^{p-1} \lambda_{1}\left|\varphi_{1}\right|^{p-1}=\lambda_{1}\left(\varepsilon \varphi_{1}\right)^{p-1} \leq\left(\varepsilon \varphi_{1}\right)^{-q}+\lambda\left(\varepsilon \varphi_{1}\right)^{\gamma}
\end{aligned}
$$

namely, $\varepsilon \varphi_{1}$ is a lower solution of (5.1). Note that $\Delta_{p}^{2}-\frac{\mu}{|x|^{2 p}}$ is monotone, then problem (5.1) has a positive solution $u_{\lambda}$ with $\varepsilon \varphi_{1} \leq u_{\lambda} \leq u_{\bar{\lambda}}$.

\section{Conclusions}

In this paper, we study a class of $p$-biharmonic equations with Hardy potential and negative exponents. We establish the dependence of the above problem on $q, \gamma, f, g$ and $\Omega$ and evaluate the extremal value of $\lambda$ related to the multiplicity of positive solutions for this problem.

\section{Funding}

This project is supported by the Natural Science Foundation of Shanxi Province (201601D011003), and the Natural Science Foundation of Shandong Province of China (ZR2017MA036).

\section{Availability of data and materials}

No data were used to support this study.

\section{Competing interests}

The authors declare that they have no competing interests.

\section{Authors' contributions}

All authors contributed equally to this work. All authors read and approved the final manuscript.

\section{Publisher's Note}

Springer Nature remains neutral with regard to jurisdictional claims in published maps and institutional affiliations.

Received: 7 June 2018 Accepted: 16 January 2019 Published online: 28 January 2019

\section{References}

1. Ansari, H., Vaezpour, S.M., Hesaaraki, M.: Existence of positive solution for nonlocal singular fourth order Kirchhoff equation with Hardy potential. Positivity 21(4), 1545-1562 (2017)

2. Aubin, J.P., Ekeland, I.: Applied Nonlinear Analysis. Pure Appl. Math. Wiley, New York (1984)

3. Benedikt, J., Drábek, P.: Estimates of the principal eigenvalue of the $p$-biharmonic operator. Nonlinear Anal. 75, 5374-5379 (2012)

4. Bhakta, M.: Entire solutions for a class of elliptic equations involving $p$-biharmonic operator and Rellich potentials J. Math. Anal. Appl. 423, 1570-1579 (2015)

5. Candito, P., Bisci, G.: Multiple solutions for a Navier boundary value problem involving the $p$-biharmonic operator. Discrete Contin. Dyn. Syst. 5, 741-751 (2012)

6. Cassani, D., do O, J., Ghoussoub, N.: On a fourth order elliptic problem with a singular nonlinearity. Adv. Nonlinear Stud. 9, 177-197 (2009)

7. Chen, Y.P., Chen, J.Q.: Existence of multiple positive weak solutions and estimates for extremal values to a class of elliptic problems with Hardy term and singular nonlinearity. J. Math. Anal. Appl. 429, 873-900 (2015)

8. Cowan, C., Esposito, P., Ghoussoub, N., Moradifam, A.: The critical dimension for a fourth order elliptic problem with singular nonlinearity. Arch. Ration. Mech. Anal. 198, 763-787 (2010)

9. Davies, E., Hinz, A.: Explicit constants for Rellich inequalities in $L^{P}(\Omega)$. Math. Z. 227, 511-523 (1998)

10. Drábek, P., Ótani, M.: Global bifurcation result for the p-biharmonic operator. Electron. J. Differ. Equ. 2001,48 (2001)

11. Gazzola, F., Grunau, H.C., Sweers, G.: Optimal Sobolev and Hardy-Rellich constants under Navier boundary conditions. Ann. Mat. Pura Appl. 189, 475-486 (2010)

12. Guan, Y.L., Zhao, Z.Q., Lin, X.L.: On the existence of positive solutions and negative solutions of singular fractional differential equations via global bifurcation techniques. Bound. Value Probl. 2016, 141 (2016) 
13. Guerra, I.: A note on nonlinear biharmonic equations with negative exponents. J. Differ. Equ. 253, 3147-3157 (2012)

14. Hao, X.A.: Positive solution for singular fractional differential equations involving derivatives. Adv. Differ. Equ. 2016 $139(2016)$

15. Huang, Y.S., Liu, X.Q.: Sign-changing solutions for $p$-biharmonic equations with Hardy potential. J. Math. Anal. Appl. 412, 142-154 (2014)

16. Lazer, A., McKenna, P.: Large amplitude periodic oscillations in suspension bridges: some new connections with nonlinear analysis. SIAM Rev. 32, 537-578 (1990)

17. Li, L.: Two weak solutions for some singular fourth order elliptic problems. Electron. J. Qual. Theory Differ. Equ. 2016, 1 (2016)

18. Li, P.R.: Generalized convolution-type singular integral equations. Appl. Math. Comput. 311, 314-323 (2017)

19. Li, P.R.: Singular integral equations of convolution type with Hilbert kernel and a discrete jump problem. Adv. Differ Equ. 2017, 360 (2017)

20. Lin, F.H., Yang, Y.S.: Nonlinear non-local elliptic equation modelling electrostatic actuation. Proc. R. Soc. Lond. Ser. A 463, 1323-1337 (2007)

21. Lin, X.L., Zhao, Z.Q.: Iterative technique for a third-order differential equation with three-point nonlinear boundary value conditions. Electron. J. Qual. Theory Differ. Equ. 2016, 12 (2016)

22. Liu, L.S., Sun, F.L., Zhang, X.G., Wu, Y.H.: Bifurcation analysis for a singular differential system with two parameters via to degree theory. Nonlinear Anal., Model. Control 22, 31-50 (2017)

23. Mao, A.M., Zhu, Y., Luan, S.X.: Existence of solutions of elliptic boundary value problems with mixed type nonlinearities. Bound. Value Probl. 2012, 97 (2012)

24. Mitidieri, E.: A simple approach to Hardy's inequalities. Math. Notes 67, 479-486 (2000)

25. Qian, A.X.: Sign solutions for nonlinear problems with strong resonance. Electron. J. Differ. Equ. 2012, 17 (2012)

26. Sreenadh, K.: On the eigenvalue problem for the Hardy-Sobolev operator with indefinite weights. Electron. J. Differ. Equ. 2002, 33 (2002)

27. Sun, F.L., Liu, L.S., Wu, Y.H.: Infinitely many sign-changing solutions for a class of biharmonic equation with $p$-Laplacian and Neumann boundary condition. Appl. Math. Lett. 73, 128-135 (2017)

28. Sun, Y.J., Li, S.J.: Some remarks on a superlinear-singular problem: estimates of $\lambda^{*}$. Nonlinear Anal. 69, 2636-2650 (2008)

29. Sun, Y.J., Wu, S.P.: An exact estimate result for a class of singular equations with critical exponents. J. Funct. Anal. 260 1257-1284 (2011)

30. Wang, X.J., Mao, A.M., Qian, A.X.: High energy solutions of modified quasilinear fourth-order elliptic equation. Bound. Value Probl. 2018, 54 (2018)

31. Wang, Y.Q., Liu, L.S.: Necessary and sufficient condition for the existence of positive solution to singular fractional differential equations. Adv. Differ. Equ. 2015, 207 (2015)

32. Xie, H.Z., Wang, J.P.: Infinitely many solutions for $p$-harmonic equation with singular term. J. Inequal. Appl. 2013, 9 (2013)

33. Xuan, B.J.: The eigenvalue problem for a singular quasilinear elliptic equation. Electron. J. Differ. Equ. 2004,16 (2004)

34. Yang, R.R., Zhang, W., Liu, X.Q.: Sign-changing solutions for $p$-biharmonic equations with Hardy potential in $\mathbb{R}^{N}$. Acta Math. Sci. 37B(3), 593-606 (2017)

35. Zhang, G.Q., Wang, X.Z., Liu, S.Y.: On a class of singular elliptic problems with the perturbed Hardy-Sobolev operator. Calc. Var. Partial Differ. Equ. 46, 97-111 (2013)

36. Zhang, Y.J.: Positive solutions of semilinear biharmonic equations with critical Sobolev exponents. Nonlinear Anal. 75 55-67 (2012)

37. Zheng, Z.W., Kong, Q.K.: Friedrichs extensions for singular Hamiltonian operators with intermediate deficiency indices. J. Math. Anal. Appl. 461, 1672-1685 (2018)

\section{Submit your manuscript to a SpringerOpen ${ }^{\circ}$ journal and benefit from:}

- Convenient online submission

- Rigorous peer review

- Open access: articles freely available online

- High visibility within the field

- Retaining the copyright to your article

Submit your next manuscript at $\boldsymbol{s p r i n g e r o p e n . c o m ~}$ 